TRANSACTIONS OF THE

AMERICAN MATHEMATICAL SOCIETY

Volume 355, Number 12, Pages 4781-4806

S 0002-9947(03)03268-9

Article electronically published on July 28, 2003

\title{
UNIQUENESS OF THE DENSITY \\ IN AN INVERSE PROBLEM FOR ISOTROPIC ELASTODYNAMICS
}

\author{
LIZABETH V. RACHELE
}

\begin{abstract}
We consider the unique determination of the density of a nonhomogeneous, isotropic elastic object from measurements made at the surface. We model the behavior of the bounded, 3-dimensional object by the linear, hyperbolic system of operators for isotropic elastodynamics. The material properties of the object (its density and elastic properties) correspond to the smooth coefficients of these differential operators. The data for this inverse problem, in the form of the correspondence between applied surface tractions and resulting surface displacements, is modeled by the dynamic Dirichlet-toNeumann map on a finite time interval. In an earlier paper we show that the speeds $c_{p / s}$ of (compressional and sheer) wave propagation through the object are uniquely determined by the Dirichlet-to-Neumann map. Here we extend that result by showing that the density is also determined in the interior by the Dirichlet-to-Neumann map in the case, for example, that $c_{p}=2 c_{s}$ at only isolated points in the object. We use techniques from microlocal analysis and integral geometry to solve this fully three-dimensional problem.
\end{abstract}

\section{INTRODUCTION AND MAIN RESUlt}

One purpose in studying dynamic inverse problems for bounded objects is to describe the material properties of the object using only measurements made at its surface. In the case of anisotropic elastodynamics the material properties of the object are modeled by 22 parameters. We may ask, then, how many of these 22 parameters may be determined by surface measurements. As a first step in addressing this question we show in [R III] that those parameters needed to describe paths of wave propagation (in the setting of rather general hyperbolic systems) are determined, to a certain extent, by surface measurements. In particular, the metrics, whose geodesics model the paths along which wave propagation occurs, are determined by surface measurements, up to pullback by a diffeomorphism. An open question is whether these metrics are determined exactly by surface measurements, and whether the other material parameters (parameters other than those needed to describe the paths of wave propagation) are also determined.

As an indication of what might be expected to be true for the dynamic inverse problem for general hyperbolic systems (using Dirichlet-to-Neumann-type data),

Received by the editors June 11, 2001.

2000 Mathematics Subject Classification. Primary 35R30.

The author was partially supported by U.S. National Science Foundation grant 9801664 (9996350). 
here we consider the remaining uniqueness question in the case of isotropic elastodynamics. In $[\mathrm{R} \mathrm{I}]$ we have shown that the (compressional and sheer) speeds $c_{p}=\sqrt{(\lambda+2 \mu) / \rho}$ and $c_{s}=\sqrt{\mu / \rho}$ of wave propagation through the object, written in terms of the elasticity parameters $\lambda$ and $\mu$ and the density $\rho$, are uniquely determined in the interior by surface measurements. In this paper we extend that result by showing that the third parameter, the density $\rho$, is also determined (in the case, for example, that the compressional wave speed $c_{p}$ is twice the sheer wave speed $c_{s}$ at only isolated points in the object). That is, we show that if two isotropic elastic objects differ only in their densities, then the surface measurements of those objects will also differ.

It is interesting that "top-order" boundary data, in the form of the boundary polarization, does not determine the (smoothly varying) density (cf. Section 3). (We have made this observation in [ $\mathrm{R} \mathrm{I}$, Section 5.3, in the case of constant wave speeds $c_{p / s}$.) Rather, it is necessary to consider "lower-order polarization". We conclude, then, that in more general settings we may expect to determine parameters other than those describing the wave paths, but that in determining other parameters, it will likely be necessary to use "lower-order" data.

A problem which has some of the features of elastodynamics is the problem solved by Rakesh and Symes in $\mathrm{Ra}-\mathrm{Sy}$. In $[\mathrm{Ra}-\mathrm{Sy}$ a disturbance that propagates through an object is modelled by a solution of the wave equation, $\left[\partial_{t}^{2}-\Delta+q(x)\right] u=$ 0 in $\Omega \times(0, T)$, where $\Omega$ is a bounded region. Rakesh and Symes show that the integral of the potential, $q(x)$, over any path along which wave energy propagates, is determined by surface measurements. Knowing these integrals of the potential is enough to recover the potential itself inside $\Omega$. In this model problem the paths along which energy propagates are straight lines, and the differential equation is scalar.

In elastodynamics, though, the paths along which wave energy propagates are not straight lines, and the elasticity equation is a system of three equations in three unknowns.

Ikehata, Nakamura, and Yamamoto [I-N-Y] use modified Carleman estimates to show that the density $\rho \in C^{2}(\bar{\Omega})$ of a bounded, isotropic elastic object in $n$ dimensional space is determined by $n$ pieces of data $\left(f_{i}, \psi_{i}, g_{i}\right)$, where $f_{i}$ is the surface displacement, $\psi_{i}$ is the initial displacement, and $g_{i}$ is the corresponding surface traction. (The $n$ initial stresses corresponding to the initial displacements $\psi_{i}$ must be uniformly linearly independent on $\bar{\Omega}$. No condition is placed on the initial velocity.) Note that $\psi_{i}$ is the initial displacement of the entire object, and so the data $\left(f_{i}, \psi_{i}, g_{i}\right)$ do not correspond to measurements that are made only at the surface of the object. In addition, the time interval for data collection must be longer than the greatest travel time of both compressional and sheer wave propagation through the object.

In comparison, in this paper we use techniques from microlocal analysis and integral geometry to determine the density $\rho \in C^{\infty}(\bar{\Omega})$, but using only surface data of the form $(f, g)$, where $f$ runs through every (sufficiently regular function modeling the) surface displacement, and $g=\Lambda_{\lambda, \mu, \rho} f$ is the corresponding surface traction. This Dirichlet-to-Neumann-type data is non-invasive or non-destructive in the sense that it corresponds to measurements that need be made only at the surface of the object. (The initial displacements and velocity are taken to be zero for each measurement.) Also, the time interval $[0, T]$ for data collection is taken 
to be the greatest travel time of just the compressional wave propagation through the object. See Uhlmann's review article [U] on inverse problems using Dirichletto-Neumann-type data.

In Sections 1.1 and 1.2 we state the main result and give a sketch of the proof. In Section 2 we present preliminaries to the proof of the main result. In Section 3 we show that the polarization at $\partial \Omega$ does not have information about $\rho$ in $\Omega$. In Section 4 we use "lower-order polarization" to show that $\rho$ is determined in $\Omega$.

1.1. Statement of the Main Result. Let $\Omega$ be a bounded region in $\mathbb{R}^{3}$ with smooth boundary. $\Omega$ represents a linearly elastic, nonhomogeneous, isotropic object if the function $\rho(x)$ representing the density is positive on $\bar{\Omega}$, if the Lamé parameters $\lambda(x)$ and $\mu(x)$, representing the elastic properties of the object, satisfy $\mu>0$ on $\bar{\Omega}$ and $3 \lambda(x)+2 \mu(x)>0$ on $\bar{\Omega}$ (the strong convexity condition), and if the vector $u(x, t)$, which represents the displacement of the object, solves the initial-boundaryvalue problem (2) associated with the hyperbolic system of operators $P$ for isotropic elastodynamics. The operator $P$ for elastodynamics is given by

$$
(P u)_{i}=\rho \partial_{t}^{2} u_{i}-\sum_{j, k, l=1}^{3} \partial_{x_{j}}\left(c_{i j k l} \partial_{x_{l}} u_{k}\right)=0, \quad i=1,2,3
$$

where $c_{i j k l}=\lambda \delta_{i j} \delta_{k l}+\mu \delta_{i k} \delta_{j l}+\mu \delta_{i l} \delta_{j k}$ is the elasticity tensor for an isotropic elastic medium. The initial-boundary-value problem is (for $0<T<\infty$ )

$$
\begin{cases}P u=0 & \text { in } \Omega \times(0, T) \\ \left.u\right|_{\partial \Omega}=f & \text { for } t \in[0, T] \\ \left.u\right|_{t=0}=0,\left.\quad\left(\partial_{t} u\right)\right|_{t=0}=0 & \text { in } \Omega\end{cases}
$$

The Dirichlet-to-Neumann map $\Lambda_{\lambda, \mu, \rho}$ models surface measurements in that it models the correspondence between a displacement $f(x, t)$ at the surface and the applied surface traction $\Lambda_{\lambda, \mu, \rho} f$ that would generate this displacement. (See [U] for a review of the use of the Dirichlet-to-Neumann map in modeling surface measurements in inverse problems.) The Dirichlet-to-Neumann map $\Lambda_{\lambda, \mu, \rho}$ is defined in terms of solutions $u$ of the initial-boundary-value problem (2) by

$$
\left(\Lambda_{\lambda, \mu, \rho} f\right)_{i}=\left.\left(\sum_{j, k, l=1}^{3} \nu_{j} c_{i j k l} \partial_{x_{l}} u_{k}\right)\right|_{\partial \Omega \times(0, T)}, \quad i=1,2,3
$$

where $\left.u\right|_{\partial \Omega \times(0, T)}=f$, and $\nu$ is the unit outer normal vector to $\partial \Omega$.

We introduce the following notation and terminology, following Sh II], Sections 3.1, 3.2, and 3.4. Given a Riemannian manifold $M$ with boundary $\partial M$ and given a point $x \in \partial M$, the second quadratic form of the boundary is defined by $\Pi(\xi, \xi)=$ $\left\langle\nabla_{\xi} \nu, \xi\right\rangle$ on $\xi \in T_{x}(\partial M)$, where $\nu=\nu(x)$ is the unit outer normal vector to the boundary. We say that the boundary is strictly convex if this form is positivedefinite for all $x \in \partial M$. We say that a compact Riemannian manifold $(M, g)$ with boundary is a compact, dissipative Riemannian manifold (CDRM) (1) if the boundary $\partial M$ is strictly convex, and (2) if there is no geodesic of infinite length in $M$.

By $T M=\left\{(x, \xi) \mid x \in M, \xi \in T_{x} M\right\}$ we denote the tangent bundle of a manifold $M$, and by $\Omega M=\{(x, \xi) \in T M|| \xi \mid=1\}$ we denote the unit tangent bundle. Let $C^{\infty}\left(\partial_{ \pm} \Omega M\right)$ be the space of smooth functions on the manifold $\partial_{ \pm} \Omega M=\{(x, \xi) \in$ $\Omega M \mid x \in \partial M$ and $\pm\langle\xi, \nu(x)\rangle \geq 0\}$, the outward/inward unit tangent bundle at the boundary. 
Given a Riemannian manifold $M$, any point $x \in M$, and a two-dimensional subspace $\sigma \subset T_{x} M$, we denote by $K(x, \sigma)$ the sectional curvature of the section $\sigma$. For $(x, \xi) \in T^{0} M=\{(x, \xi) \in T M \mid \xi \neq 0\}$ we set $K(x, \xi)=\sup _{\{\xi \in \sigma\}} K(x, \sigma)$ and $K^{+}(x, \xi)=\max \{0, K(x, \xi)\}$. For a $\operatorname{CDRM}(M, g)$ we define

$$
k^{+}(M, g)=\sup _{(x, \xi) \in \partial_{-} \Omega M} \int_{0}^{\tau_{+}(x, \xi)} t K^{+}\left(\gamma_{x, \xi}(s), \dot{\gamma}_{x, \xi}(s)\right) d s,
$$

where $\gamma_{x, \xi}:\left[0, \tau_{+}(x, \xi)\right]$ is the maximal geodesic in $M \cup \partial M$ satisfying the initial (i.e., entry) conditions $\left(\gamma_{x, \xi}(0), \dot{\gamma}_{x, \xi}(0)\right)=(x, \xi) \in \partial_{-} \Omega M$.

Our main result is the unique determination of the density $\rho$ in $\Omega$ by surface measurements:

Theorem 1 (Main Result). Let $\Omega$ be a bounded region in $\mathbb{R}^{3}$ with smooth boundary. Suppose that the coefficients $\lambda_{i}, \mu_{i}, \rho_{i} \in C^{\infty}(\bar{\Omega})$ satisfy $\rho_{i}, \mu_{i}>0,3 \lambda_{i}+2 \mu_{i}>0$ (strong convexity) for $i=1,2$. In addition, suppose that (i) the metrics $g_{p}^{i}=$ $\left(\rho_{i} /\left(\lambda_{i}+2 \mu_{i}\right)\right) e, g_{s}^{i}=\left(\rho_{i} / \mu_{i}\right) e$, where $e$ is the Euclidean metric, do not give rise to caustics, (ii) the geodesics of $g_{p / s}^{i}$ all exit $\Omega$ before some time $T<\infty$, and (iii) the geodesics of $g_{p / s}^{i}$ do not graze the boundary. Finally, suppose that $\left(\Omega, g_{p}^{i}\right) \quad$ is a compact, dissipative Riemannian manifold with $3 k^{+}\left(M, g_{p}^{i}\right)<1$. Then $\Lambda_{\lambda_{1}, \mu_{1}, \rho_{1}}=\Lambda_{\lambda_{2}, \mu_{2}, \rho_{2}}$ on $\partial \Omega \times(0, T)$ implies

$$
\rho_{1}=\rho_{2}
$$

in the closure of an open set $\mathcal{C}$ in $\Omega$ (defined in (56) ) where $\lambda_{i} \neq 2 \mu_{i}$. In particular, if $\lambda_{i}=2 \mu_{i}, \quad i=1,2$, only at isolated points in $\bar{\Omega}$, then $\rho_{1}=\rho_{2}$ on $\bar{\Omega}$.

Remark 1 . The condition $3 k^{+}(M, g)<1$ is satisfied if the curvature of $\left(\Omega,\left(1 / c_{p}^{2}\right) e\right)$ is bounded above and is small enough along each geodesic, relative to the length of the geodesic. For example, in the case that the wave speed $c_{p}$ is constant, the manifold $\left(\Omega,\left(1 / c_{p}^{2}\right) e\right)$ is flat (zero curvature), and thus satisfies $3 k^{+}(M, g)<1$. Also, in the case that $\left(\Omega,\left(1 / c_{p}^{2}\right) e\right)$ has negative curvature (e.g., the hyperbolic disk), the condition $3 k^{+}(M, g)<1$ is easily satisfied due to the fact that $K\left(\gamma_{x, \xi}(s), \dot{\gamma}_{x, \xi}(s)\right)<0$, and so $K^{+}\left(\gamma_{x, \xi}(s), \dot{\gamma}_{x, \xi}(s)\right) \equiv 0$.

Other upper bound conditions on $k^{+}(M, g)$ may be used to describe classes of CDRM. For example (cf. [Sh II], Section 3.4), if a CDRM $(M, g)$ has $k^{+}(M, g)<1$, then $M$ is diffeomorphic to the ball, and $(M, g)$ is simple; that is, (i) the boundary is strictly convex, and (ii) any two points $x, y \in M$ are joined by a unique geodesic in a way that depends smoothly on $x$ and $y$ (that is, the mapping $\exp _{x}^{-1}: M \rightarrow T_{x} M$ is smooth).

Remark 2. In this paper we consider $\left(\Omega, g_{p}\right)$ with $g_{p}=\left(1 / c_{p}^{2}\right) e$; so we find

$$
\begin{aligned}
& K\left(\gamma_{x, \xi}(s), \dot{\gamma}_{x, \xi}(s)\right) \\
& =\sup _{\vec{n}} \frac{1}{c_{p}^{2}}\left[\vec{n}^{t}\left(\log c_{p}\right)_{x x} \vec{n}+\dot{\gamma}_{x, \xi}(s)^{t}\left(\log c_{p}\right)_{x x} \dot{\gamma}_{x, \xi}(s)\right. \\
& \left.\quad-(1 / 2)\left(\left[\dot{\gamma}_{x, \xi}(s) \cdot\left(\log c_{p}\right)_{x}\right]^{2}+\left[\vec{n} \cdot\left(\log c_{p}\right)_{x}\right]^{2}+\left|\left(\log c_{p}\right)_{x}\right|^{2}\right)\right]
\end{aligned}
$$

where the supremum is taken over $\vec{n}$ with $\vec{n}$ orthogonal to $\dot{\gamma}_{x, \xi}(s)$. It follows that $3 k^{+}(M, g)<1$ is satisfied, for example, if $\log c_{p}$ is concave on $\Omega$ (that is, if 
$\log c_{p}(\alpha x+[1-\alpha] y) \geq \alpha \log c_{p}(x)+[1-\alpha] \log c_{p}(y)$ for any constant $\alpha \in[0,1]$ and $x, y \in \Omega)$.

Remark 3. The result of Rakesh and Symes Ra-Sy for the wave equation $\left[\partial_{t}^{2}-\Delta+q(x)\right] u=0$ applies to media with a constant wave speed and so curvature $K(x, \xi) \equiv 0$; that is, $k^{+}(M, g) \equiv 0$. In this paper we consider a generalization to elastodynamics and to the case of media with $k^{+}(M, g)$ not necessarily zero. (See the introduction of this article for a description of Rakesh and Symes' result.)

Remark 4. Conditions (i)-(iii) of Theorem 1 are imposed in order to apply [R I], Theorem 1, which finds that the wave speeds $c_{p / s}^{i}$ are determined in $\Omega$. This result is preliminary to proving uniqueness of the density in $\Omega$ (cf. Section 2.5). The conditions that $\left(\Omega, g_{p}^{i}\right)$ be a compact, dissipative Riemannian manifold with $3 k^{+}\left(M, g_{i}\right)<1$ are imposed in Theorem 1 in order to apply Sharafutdinov's inversion of the ray transform for a tensor field (cf. Section 4.2). We restrict the conclusion of Theorem 1 to the region $\mathcal{C}$ due to the need to guarantee uniqueness for the fourth-order, linear, elliptic differential equation (53). The singular points of (53), that is, the zeros of the leading coefficient $\gamma$, are the points in $\bar{\Omega}$ where $\lambda_{i}=2 \mu_{i}$, i.e., where $c_{p}=2 c_{s}$. (Compare $c_{p} \neq 2 c_{s}$, i.e., $c_{p}^{2} \neq 4 c_{s}^{2}$, with the strong convexity condition $3 \lambda+2 \mu>0$, i.e., $3 c_{p}^{2}>4 c_{s}^{2}$.)

1.2. Sketch of the Proof. In elastodynamics, wave energy from a disturbance propagates through the medium along paths that are determined by the density and elasticity. In fact, these paths are, in the isotropic case, geodesics, curves along which travel time is minimized; so they are given in terms of the wave speeds $c_{p}$ and $c_{s}$ of the medium, which, in turn, are given in terms of the density $\rho(x)$ and elastic parameters, $\lambda(x)$ and $\mu(x)$. We derive explicit descriptions of these wave paths (cf. Section 2.3) in terms of $\lambda, \mu$ and $\rho$.

We then apply the boundary determination (Theorem 2.1): if $\Lambda_{\lambda_{1}, \mu_{1}, \rho_{1}}=$ $\Lambda_{\lambda_{2}, \mu_{2}, \rho_{2}}$, then it follows that $\partial_{\nu}^{j} \lambda_{1}=\partial_{\nu}^{j} \lambda_{2}, \partial_{\nu}^{j} \mu_{1}=\partial_{\nu}^{j} \mu_{2}$, and $\partial_{\nu}^{j} \rho_{1}=\partial_{\nu}^{j} \rho_{2}$ on $\partial \Omega, j=0,1, \ldots$, where $\partial_{\nu}$ is the normal derivative at $\partial \Omega$. We conclude in [R I] that the object may be viewed as being embedded in a medium of infinite extent with smoothly varying density and elasticities at the interface of the surface of the object and the surrounding medium. In particular, we show that the displacement of the medium is uniquely determined outside the object by the Dirichlet-to-Neumann map (cf. Section 2.1). In [R I] we conclude that asymptotic expansions of the displacement of the object and the surrounding medium may be written, in this case, in terms of global Fourier integral operators on $\mathbb{R}^{3}$. We have applied results on the propagation of singularities and results from integral geometry to conclude in [R I] that the compressional and sheer wave speeds $c_{p / s}$ associated with the medium are determined in $\Omega$ by the Dirichlet-to-Neumann map.

We now consider the determination of $\rho$ in the interior. We remark that a consequence of the determination of the density $\rho$, given the determination of the wave speeds $c_{p}=\sqrt{(\lambda+2 \mu) / \rho}$ and $c_{s}=\sqrt{\mu / \rho}$, will be that each of the three parameters $\lambda, \mu$, and $\rho$ is determined.

In Section 3 we show that polarization boundary data contains no information about the (smooth) density in the interior.

In Section 4 we show that the boundary values of "lower-order polarization" do determine $\rho$ in $\Omega$. In particular, we consider the decomposition of the displacement vector as the sum of terms, graded by the strength of their singularities. In fact, 
the displacement vector is represented in terms of Fourier integral operators (FIOs) acting on initial $(t=0)$ data (an FIO for each of the forward/backward $p / s$-waves). The amplitudes of the FIOs are written as the sum of terms $a_{0}, a_{-1}, a_{-2}, \ldots$ of homogeneity $0,-1,-2, \ldots$ with respect to the frequency. The top-order terms $a_{0}$ describe the most singular behavior of the displacement vector (the polarization). The lower-order terms $a_{-1}, a_{-2}, \ldots$ describe less singular behavior. For the forward $p$-wave FIO we write $a_{0}=\alpha_{0} N$, with $N$ the (unit) vector in the direction of the gradient of the phase, i.e., parallel to the direction of displacement of the $p$ wave, and we write $a_{-1}=h_{1}+\alpha_{-1} N$, with $h_{-1}$ orthogonal to $N$. (Similarly, $a_{-J}=h_{-J}+\alpha_{-J} N$.) We refer to the term $\alpha_{-1} N$ as the lower-order polarization.

The $\alpha_{-J}$ solve ODEs (the transport equations) along the bicharacteristics of $P$. Therefore, we write the (lower-order) polarization in terms of line integrals along rays in order to derive a ray transform defined on ray segments between boundary points of the object. It follows from the fact that (lower-order) polarization is determined at the boundary (and outside the object), that this ray transform is determined by the Dirichlet-to-Neumann map. We invert the ray transform by applying results of Sharafutdinov. It follows that the tensor field appearing in the integrand of the ray transform is determined (modulo its potential part) at each interior point. We filter the integrand (via use of the Saint-Venant operator) to derive a fourth-order, linear, elliptic partial differential equation $E \beta^{-}=0$ which has solution involving the difference between $\rho_{1}$ and $\rho_{2}$. Then, applying uniqueness results, we conclude that the density is, in fact, determined in a part of the interior described in terms of the topology of the singular points of $E$.

\section{Preliminaries to the proof of the main Result}

2.1. Embedding the object in an unbounded elastic domain. The author shows in [ $\mathrm{R} \mathrm{II}]$ that $\lambda, \mu, \rho$, and their normal derivatives are determined to infinite order by the Dirichlet-to-Neumann map at the boundary. In fact,

Theorem 2.1 (Rachele [R II], Theorem 1). Let $\Omega$ be a bounded region in $\mathbb{R}^{3}$ with smooth boundary. Suppose that the coefficients $\lambda_{i}, \mu_{i}, \rho_{i} \in C^{\infty}(\bar{\Omega})$ satisfy $\rho_{i}, \mu_{i}>$ 0 , and $3 \lambda_{i}+2 \mu_{i}>0$ (strong convexity) on $\bar{\Omega}$. Then $\Lambda_{\lambda_{1}, \mu_{1}, \rho_{1}}=\Lambda_{\lambda_{2}, \mu_{2}, \rho_{2}}$ on $\partial \Omega \times(0, T)$ for any $T>0$ implies

$$
\partial_{\nu}^{j} \lambda_{1}=\partial_{\nu}^{j} \lambda_{2}, \quad \partial_{\nu}^{j} \mu_{1}=\partial_{\nu}^{j} \mu_{2}, \quad \partial_{\nu}^{j} \rho_{1}=\partial_{\nu}^{j} \rho_{2} \quad \text { on } \partial \Omega
$$

for $j=0,1, \ldots$

Theorem 2.1 is applied in [ $\mathrm{R} \mathrm{I}$, Section 3 , to extend $\lambda, \mu$, and $\rho$ smoothly to all of $\mathbb{R}^{3}$ so that each is determined by $\Lambda_{\lambda, \mu, \rho}$ outside $\Omega$. As in [S-U], we then approach the question of uniqueness in the interior from the point of view of the Cauchy problem for elastodynamics on $\mathbb{R}^{3} \times(0, T)$,

$$
\left\{\begin{aligned}
P U & =0 & & \text { on } \mathbb{R}^{3} \times(0, T), \\
\left.U\right|_{t=0} & =\psi_{0} & & \text { on } \mathbb{R}^{3}, \\
\left.\partial_{t} U\right|_{t=0} & =\psi_{1} & & \text { on } \mathbb{R}^{3},
\end{aligned}\right.
$$

rather than the initial-boundary-value problem (2) on $\Omega \times(0, T)$. We may view the elastic object as being embedded in a larger (unbounded) elastic domain, due to $[\mathrm{R} \mathrm{I}$, Theorem 3.1, which we state below. In $[\mathrm{R} \mathrm{I}$, Theorem 3.1, we show that the 
displacement vectors $U$ that solve the Cauchy problem (3) on $\mathbb{R}^{3}$ for the operator $P$ for isotropic elastodynamics are determined outside $\Omega$ by the Dirichlet-to-Neumann map, given fixed boundary data and fixed initial data that is supported outside $\Omega$.

Theorem 2.2 (Rachele $[\mathrm{R} \mathrm{I}$, Theorem 3.1). Suppose that the conditions of Theorem 2.1 hold. Let $P_{j}=P\left(\lambda_{j}, \mu_{j}, \rho_{j}\right), j=1,2$, be the hyperbolic system of operators of the form (1), but defined on all of $\mathbb{R}^{3}$, and suppose that $\lambda_{1}=\lambda_{2}, \mu_{1}=$ $\mu_{2}, \quad \rho_{1}=\rho_{2}$ outside $\Omega$. In addition, suppose that $U_{j}(j=1,2)$ solves the Cauchy problem (3) on $\mathbb{R}^{3} \times(0, T)$, where $\left(\operatorname{supp} \psi_{k}\right) \cap \bar{\Omega}=\emptyset$ for $k=0,1$, and

$$
\left.U_{1}\right|_{\partial \Omega \times(0, T)}=\left.U_{2}\right|_{\partial \Omega \times(0, T)} .
$$

Then $\Lambda_{\lambda_{1}, \mu_{1}, \rho_{1}}=\Lambda_{\lambda_{2}, \mu_{2}, \rho_{2}}$ on $(0, T)$ implies

$$
U_{1}(x, t)=U_{2}(x, t) \text { outside } \Omega, \text { for } t \in(0, T) .
$$

2.2. Constructing Fourier integral operator representations of the elastic displacement wave. In [ R I] we construct asymptotic expansions of certain solutions $U_{j}$ of the Cauchy problem (3). These solutions $U_{j}$ have wave front set that is minimal in the sense that it is generated by initial data with wave front set at a single point along a single ray (cf. [R I], Lemma 4.3). The construction of these asymptotic expansions is central to the main result of this paper and is summarized below.

We construct solutions $U$ of the Cauchy problem (3) on $\mathbb{R}^{3} \times(0, T)$ by writing

$$
U=E_{0} \psi_{0}+E_{1} \psi_{1}
$$

in terms of the initial data $\psi_{0}, \psi_{1}$ and solution operators $E_{0}, E_{1}\left(t, x, D_{x}\right)$. These solution operators solve

$$
\left\{\begin{array}{rlll}
P_{c} E_{\underline{k}} & \equiv 0 & \text { on } \mathbb{R}^{3} \times(0, T) \quad \text { for } \underline{k}=0,1, \\
\left.E_{0}\right|_{t=0} & \equiv I, & \left.E_{1}\right|_{t=0} \equiv 0,
\end{array}\right\} \text { mod smoothing, }
$$

and, in fact, may be written in terms of the Fourier integral operator Ansatz

$$
\left(E_{\underline{k}} \mu\right)_{\underline{l}}=\sum_{p / s, \underline{m}, \pm} \int e^{i \varphi_{p / s}^{ \pm}} \quad e_{p / s, \pm}^{\underline{l}, \underline{m}}(\underline{k}) \widehat{\mu}_{\underline{m}}(\eta) d \eta, \quad\left(\begin{array}{c}
\underline{k}=0,1 \\
\underline{l}=1,2,3
\end{array}\right) .
$$

In $\left[\mathrm{R} \mathrm{I}\right.$, Section 2, we derive conditions on the phase functions $\varphi_{p / s}^{ \pm}(t, x, \eta)$ and amplitudes $e_{p / s}^{\underline{l}, \underline{m}}(\underline{k})(t, x, \eta)$ that appear in this oscillatory integral representation (5) of the solution operators $E_{\underline{k}}$. We find, for example, that the phase functions $\varphi_{p / s}^{ \pm}$are homogeneous of order 1 in $|\eta|$ and solve the eikonal equations $\operatorname{det} p\left(t, x, \partial_{t, x} \varphi_{p / s}^{ \pm}\right)=$ 0, i.e.,

$$
\partial_{t} \varphi_{p / s}^{ \pm}=\mp c_{p / s}\left|\nabla_{x} \varphi_{p / s}^{ \pm}\right|
$$

The eikonal equations are noncharacteristic, first-order nonlinear equations which can be solved by Hamilton-Jacobi theory (cf., for example, [Gr-Sj]), with initial values

$$
\left.\varphi_{p / s}^{ \pm}\right|_{t=0}=x \cdot \eta
$$

given in terms of the parameter $\eta \in \mathbb{R}^{3} \backslash 0$. It follows that the gradient of $\varphi_{p / s}$ takes on the following simple form at $t=0$ :

$$
\left.\nabla_{x} \varphi_{p / s}^{ \pm}\right|_{t=0}=\eta \text {. }
$$


The amplitudes $e_{p / s}(\underline{\mathrm{k}})$ can be written in the form

$$
\left(e_{p / s}^{\cdot, \underline{m}}(\underline{k})\right)_{J}=\left(h_{p / s}^{\cdot, \underline{m}}\right)_{J}+\left\{\begin{array}{c}
\left(\alpha^{\underline{m}}\right)_{J} N \\
\left(\alpha_{1}^{\underline{m}}\right)_{J} N_{1}+\left(\alpha^{\frac{m}{2}}\right)_{J} N_{2}
\end{array}\left(\begin{array}{c}
J=0,-1, \ldots \\
\underline{k}=0,1 \\
p / s, \pm \\
\underline{m}=1,2,3
\end{array}\right)\right.
$$

where $\left(e_{p / s}(\underline{k})\right)_{J}$ is the part of $e_{p / s}(\underline{k})$ that is homogeneous of order $J$ in $|\eta|$. In addition, the $\left(\alpha^{\underline{m}, \pm}\right)_{J}(k)$ and $\left(\alpha_{j}^{\underline{m}, \pm}\right)_{J}(\underline{k})(t, x, \eta)$ are scalars, $\left(h_{p}^{\cdot, \underline{m}}\right)_{0}=0, \quad\left(h_{p / s}^{\cdot,}, \underline{m}\right)_{J}$ is in the cokernel of $p\left(t, x, \partial_{t, x} \varphi_{p / s}\right)$ for $J=-1,-2, \ldots$, and $N$ and $N_{1}, N_{2}$ form an orthonormal basis of the kernel of $p\left(t, x, \partial_{t, x} \varphi\right)$ for $\varphi=\varphi_{p}$ and $\varphi=\varphi_{s}$, respectively. That is, $N=\frac{\nabla_{x} \varphi_{p}}{\left|\nabla_{x} \varphi_{p}\right|}$, and $N_{1}, N_{2}, \frac{\nabla_{x} \varphi_{s}}{\left|\nabla_{x} \varphi_{s}\right|}$ are mutually orthonormal.

The amplitudes solve algebraic equations:

$$
\begin{aligned}
& p\left(t, x, \partial_{t, x} \varphi_{p / s}^{ \pm}\right)\left(e_{p / s}(\underline{k})\right)_{J-1} \\
& \quad=B_{p / s}\left(e_{p / s}(\underline{k})\right)_{J}+C_{p / s}\left(e_{p / s}(\underline{k})\right)_{J+1}, \quad\left(\begin{array}{c}
\underline{k}=0,1 \\
p / s, \pm \\
J=0,-1, \ldots
\end{array}\right)
\end{aligned}
$$

where

$$
\begin{aligned}
p(t, x, \tau, \xi) & =\left(-\rho \tau^{2}+\mu|\xi|^{2}\right) I+(\lambda+\mu)(\xi \otimes \xi) \\
& =-\rho\left[\left(\tau^{2}-c_{s}^{2}|\xi|^{2}\right) I-\left(c_{p}^{2}-c_{s}^{2}\right)(\xi \otimes \xi)\right]
\end{aligned}
$$

is the principal symbol of $P(t, x, D), \quad p_{1}(t, x, \tau, \xi)$ is the sum of the lower-order terms in the symbol of $P$,

$$
\begin{array}{r}
B_{p / s} M=i \partial_{\tau, \xi} p\left(t, x, \partial_{t, x} \varphi_{p / s}^{ \pm}\right) \cdot \partial_{t, x} M+i\left(i p_{1}\right)\left(t, x, \partial_{t, x} \varphi_{p / s}^{ \pm}\right) M \\
+\frac{i}{2} \sum_{|\alpha|=2} \sum_{l=1}^{3}\left(\partial_{\tau, \xi}^{\alpha} p^{i l}\right)\left(t, x, \partial_{t, x} \varphi_{p / s}^{ \pm}\right) \cdot\left(\partial_{t, x}^{\alpha} \varphi_{p / s}^{ \pm}\right) M^{l m} \\
C_{p / s} M=\partial_{\tau, \xi}\left(i p_{1}\right)\left(t, x, \partial_{t, x} \varphi_{p / s}^{ \pm}\right) \cdot \partial_{t, x} M \\
\quad+\frac{1}{2} \sum_{|\alpha|=2} \sum_{l=1}^{3}\left(\partial_{\tau, \xi}^{\alpha} p^{i l}\right)\left(t, x, \partial_{t, x} \varphi_{p / s}^{ \pm}\right) \cdot \partial_{t, x}^{\alpha} M^{l \underline{m}}
\end{array}
$$

and, for ease of notation, we set

$$
\left(e_{p / s}(\underline{k})\right)_{1}=0 .
$$

In Section 4.1 we reduce the compatibility conditions for (9),

$$
N_{p / s}\left[B_{p / s}\left(e_{p / s}(\underline{k})\right)_{J}+C_{p / s}\left(e_{p / s}(\underline{k})\right)_{J+1}\right]=0 \quad\left(\begin{array}{c}
J=0,-1, \ldots \\
p / s, \pm \\
\underline{k}=0,1
\end{array}\right)
$$

to the transport equations, first-order ordinary differential equations in $\left(\alpha^{\underline{m}}\right)_{J}$ along $p$-wave characteristics of $P$, and systems of two first-order ordinary differential equations in $\left(\alpha_{1}^{\frac{m}{1}}\right)_{J}$ and $\left(\alpha \frac{m}{2}\right)_{J}$ along $s$-wave characteristics of $P$. Here $N_{p}=N$ spans ker $p\left(t, x, \partial_{t, x} \varphi_{p}\right)$ and $N_{s} \in\left\{N_{1}, N_{2}\right\}$, which span $\operatorname{ker} p\left(t, x, \partial_{t, x} \varphi_{s}\right)$. It follows from (9) that the $h$ and $\alpha$ defining the $e(\underline{k})$ at one level are given in terms of the $e(\underline{k})$ from the two previous levels. We can therefore choose the lower-order $\left(h_{p / s}^{\cdot,},{ }^{,}{ }^{ \pm}\right)_{J-1}$ to solve the (algebraic) equations (9), and we can define the $\left(\alpha^{\underline{m}}\right)_{J-1}$ and $\left(\alpha_{j}^{\underline{m}}\right)_{J-1}$ to be solutions of the transport equations for $J-1$. 
Assuming that the initial data $\psi_{0}, \psi_{1}$ are both homogeneous of order zero in $|\eta|$, and taking

$$
N_{1}^{+}=N_{1}^{-} \quad \text { and } \quad N_{2}^{+}=N_{2}^{-} \quad \text { at } t=0
$$

we find that there is a unique choice at $t=0$ for the scalars $\left(\alpha^{m}\right)_{J}$ and $\left(\alpha_{i}^{m}\right)_{J}$ for each $J=0,-1, \ldots$ (see [R I], Section 2.4). In the case $J=0$, for example, we have

$$
\begin{aligned}
& \left(\alpha^{\underline{m}, \pm}\right)_{0}=\frac{1}{2} e_{\underline{m}} \delta_{\underline{k} 0} \cdot N=\frac{1}{2} \frac{\eta_{\underline{m}}}{|\eta|} \delta_{\underline{k} 0}, \\
& \left(\alpha_{i}^{\underline{m}, \pm}\right)_{0}=\frac{1}{2} e_{\underline{m}} \delta_{\underline{k} 0} \cdot N_{i}=\frac{1}{2}\left(N_{i}\right)_{\underline{m}} \delta_{\underline{k} 0}, \quad i=1,2 .
\end{aligned}
$$

It follows that the principal term $\left(e_{p,+}\right)_{0}($ at $t=0)$ of $e_{p,+}=e_{p,+}(0)(0, x, \eta)$ is given by

$$
\left(e_{p,+}\right)_{0}=\left(\left(\alpha^{1}\right)_{0} N, \quad\left(\alpha^{2}\right)_{0} N, \quad\left(\alpha^{3}\right)_{0} N\right)=\frac{1}{2} \frac{\eta}{|\eta|} \otimes \frac{\eta}{|\eta|}
$$

since $($ at $t=0) \nabla_{x} \varphi_{p}(0, x, \eta)=\eta$ by (17) and so (at $\left.t=0\right) N=\frac{\eta}{|\eta|}$ by (18). (Recall that $\left.(v \otimes w)_{i j}=v_{i} w_{j}.\right)$ That is, $\left.\left(e_{p,+}\right)_{0}\right|_{t=0}=\frac{1}{2} \operatorname{Proj}_{\eta}$ where $\operatorname{Proj}_{\eta}=\frac{\eta}{|\eta|} \otimes \frac{\eta}{|\eta|}$ is the projection onto the space spanned by $\eta$. Similarly, $\left.e_{s, \pm}(0)\right|_{t=0}=\frac{1}{2} \operatorname{Proj}_{\eta^{\perp}}$, where $\operatorname{Proj}_{\eta^{\perp}}=I-\frac{\eta}{|\eta|} \otimes \frac{\eta}{|\eta|}$ is the projection onto the space orthogonal to $\eta$.

2.3. Modeling wave paths by bicharacteristics of $P$. We apply Dencker's De] propagation of singularities result ([De], Theorem 4.2; see also [De], Definition 4.1 and equation (3.4)) for systems of real principal type (cf. [R I], Section 4.1) to conclude that singularities propagate along bicharacteristics of $P$. That is, bicharacteristic curves of $P$ can be used to model the paths along which wave propagation occurs. Bicharacteristic curves, for flow out of $t=0$, are integral curves $(t(s), x(s), \tau(s), \xi(s))$ of Hamilton vector fields $V_{H_{p / s}^{ \pm}}$, with the restriction that the (null) bicharacteristic curves lie in the characteristic set $\{(t, x, \tau, \xi) \mid$ det $p(t, x, \tau, \xi)=0\}$ of $P$. The Hamilton vector field $V_{H}$ of any (smooth) Hamiltonian function $H(t, x, \tau, \xi)$ is defined by

$$
V_{H}=\left(\partial_{\tau} H\right) \partial_{t}+\left(\nabla_{\xi} H\right) \cdot \nabla_{x}-\left(\partial_{t} H\right) \partial_{\tau}-\left(\nabla_{x} H\right) \cdot \nabla_{\xi}
$$

The Hamiltonians

$$
H_{p / s}^{ \pm}(t, x, \tau, \xi)=\tau \pm c_{p / s}|\xi|
$$

are factors of the determinant of the principal symbol $p(t, x, \tau, \xi)$ of $P$ (cf. (10) ). (See Hörmander [Hö] for a description of bicharacteristics in terms of the Hamilton flow in the scalar case; replace $H_{p}$ by $H_{\operatorname{det} \sigma_{\mathrm{pr}}(P)}$ in the proof of Hörmander's Theorem 6.4.3 in the case that $P$ is a system.) That is, bicharacteristics have direction

$$
\frac{d t}{d s}=\frac{1}{c_{p / s}}, \quad \frac{d x}{d s}=\frac{ \pm \xi}{|\xi|}, \quad \frac{d \tau}{d s}=0, \frac{d \xi}{d s}=\mp\left(\log c_{p / s}\right)_{x}|\xi|
$$

and satisfy $\operatorname{det} p(t, x, \tau, \xi)=-\rho^{3}\left(\tau^{2}-c_{p}^{2} \mid \xi^{2}\right)\left(\tau^{2}-c_{s}^{2} \mid \xi^{2}\right)^{2}=0$.

The component $x(s)$ of the bicharacteristic curves corresponds to position along the wave path, and is in fact a geodesic with respect to the metric $g_{i j}=\frac{1}{c_{p / s}^{2}} \delta_{i j}$ (cf. [R I], Proposition 4.1). The component $t(s)$ of the bicharacteristics of $P$ corresponds to the travel time of the wave, i.e., geodesic distance. 
Given the eikonal equations $\operatorname{det} p\left(t, x, \partial_{t, x} \varphi_{p / s}\right)=0$ (cf. (6) $)$, we conclude that wave paths are described in terms of the phase functions $\varphi_{p / s}$ by bicharacteristics $(t, x, \tau, \xi)$ with $\tau=\partial_{t} \varphi_{p / s}$ and $\xi=\nabla_{x} \varphi_{p / s}$.

\subsection{The bicharacteristics of $P$ are determined outside $\Omega$ by the Dirichlet- to-Neumann map. We denote by}

$$
\Gamma_{p / s, \pm}=\Gamma_{p / s, \pm}(\underline{x}, \underline{\xi})
$$

the (forward or backward) null bicharacteristic of $P$ that passes through $(t, x, \tau, \xi)=$ $\left(0, \underline{x}, \mp c_{p / s}|\underline{\xi}|, \underline{\xi}\right)$ where $\underline{s}=0, \underline{x}=x(\underline{s}) \notin \Omega, \underline{\xi}=\xi(\underline{s})$.

Theorem 2.3 (Rachele [R I], Theorem 4.2). Suppose that the conditions of Theorem 2.1 hold. Suppose further that the coefficients $\lambda_{i}, \mu_{i}, \rho_{i}$ do not give rise to caustics for $i=1,2$. Then for any $\underline{x} \notin \bar{\Omega}$ and $\underline{\xi} \in \mathbb{R}^{3} \backslash 0$ the bicharacteristics $\Gamma_{p / s, \pm}(\underline{x}, \underline{\xi})$ are determined by $\Lambda_{\lambda, \mu, \rho}$ outside $\Omega$ for $t \in(0, T)$.

Proof. For $\left(\lambda_{1}, \mu_{1}, \rho_{1}\right)$ and $\left(\lambda_{2}, \mu_{2}, \rho_{2}\right)$ with $\Lambda_{\lambda_{1}, \mu_{1}, \rho_{1}}=\Lambda_{\lambda_{2}, \mu_{2}, \rho_{2}}$ we construct solutions $U_{j}$ of the Cauchy problem (3) as in the proof of [ $\mathrm{R} \mathrm{I}$ ], Theorem 3.1, with initial data $\psi^{0}$ and $\psi^{1}$ (to be chosen later) supported outside $\Omega$. By [ $\mathrm{R} \mathrm{I}$, Theorem 3.1 , these $U_{j}$ are determined outside $\Omega$, and so their wave front sets agree outside $\Omega$ :

$$
\text { WF } U_{1}=\text { WF } U_{2} \quad \text { outside } \bar{\Omega} \text {. }
$$

We now choose initial data $\psi_{0}$ and $\psi_{1}$ with "minimal" wave front set, so that the wave front sets of the corresponding solutions $U_{1}$ and $U_{2}$ will consist of only a few null bicharacteristic strips of $P$. In fact, we choose

$$
\psi_{0}=h(x) \xi_{0} \quad \text { and } \quad \psi_{1}=0
$$

where $\xi_{0} \cdot \underline{\xi} \neq 0, \quad \xi_{0} \times \xi \neq 0$, and $h(x)$ is a scalar function on $\mathbb{R}^{3}$ with WF $h(x)=$ $\{(\underline{x}, \alpha \underline{\xi}): \alpha>0\}$. (See [R I], Remark 4.1 on the existence of such distributions $h(x).)^{-}$

Given these initial values, we write the components of $U_{j}$ as $U_{j}^{l}=\Sigma_{p / s, \pm} E_{p / s, \pm}^{l} h$, $l=1,2,3$, where $E_{p / s, \pm}^{l}\left(t, x, D_{x}\right)$ is the scalar Fourier integral operator (cf. (4) and (5)) given by

$$
E_{p / s, \pm}^{l}\left(t, x, D_{x}\right) h=\int e^{i \varphi_{p / s, \pm}(t, x, \eta)}\left[e_{p / s, \pm}^{l, \cdot}(0) \xi_{0}\right] \hat{h}(\eta) d \eta .
$$

Then, given the initial values (7) for the phase, we describe the wave front set of the pseudodifferential operators $\left.E_{p / s, \pm}^{l}\right|_{t=0}$ acting on $h$. In particular, we observe that the principal symbol of $\left.E_{p / s, \pm}\right|_{t=0}$ is given by $\left.\left(E_{p / s, \pm}\right)_{0}(t, x, \eta)\right|_{t=0}=$ $\left.\left(e_{p / s, \pm}\right)_{0}(j, 0)(t, x, \eta) \xi_{0}\right|_{t=0}$, which is $\frac{1}{2} \operatorname{Proj}_{\eta} \xi_{0}$ in the case of $p$-waves, and $\frac{1}{2} \operatorname{Proj}_{\eta^{\perp}} \xi_{0}$ in the case of $s$-waves by (12) and the text that follows. Therefore, by the choice of $\xi_{0},(\underline{x}, \underline{\xi}) \in \mathrm{WF}\left(\left.E_{p / s, \pm}^{l}\right|_{t=0} h\right) \neq \emptyset$ for some $l \in\{1,2,3\}$. In addition, by $[\mathrm{Eg}]$, Corollary 4.1, p. 141, for example,

$$
\left.\mathrm{WF}\left(\left.E_{p / s, \pm}^{l}\right|_{t=0} h\right) \subseteq \pi_{(x, \xi)}\left[\mathrm{WF}\left(E_{p / s, \pm}^{l} h\right)\right]\right|_{t=0},
$$

and so

$$
\mathrm{WF}\left(E_{p / s, \pm}^{l} h\right) \text { is not empty for some } l \in\{1,2,3\} \text {. }
$$


On the other hand, WF $\left(E_{p / s, \pm}^{l} h\right)$ can be written in terms of the canonical transformation associated with the scalar Fourier integral operator $E_{p / s, \pm}^{l}$ :

$$
\begin{aligned}
\mathrm{WF}\left(E_{p / s, \pm}^{l} h\right) & \subseteq C_{p / s, \pm} \circ\{(\underline{x}, \underline{\xi}): \beta>0\} \\
& =\text { conic-closure }\left(\Gamma_{p / s, \pm}^{j}(\underline{x}, \underline{\xi})\right)
\end{aligned}
$$

by $[\operatorname{Tr}$, Theorem 4.1, for example.

We note that the wave front set of $U_{j}$ is the union of the wave front sets of its components, and that the bicharacteristic strips $\Gamma_{p / s, \pm}$ are disjoint. Also, by Dencker [De], Theorem 4.2, (see also Section 6.1), the wave front set of $U_{j}$ is invariant under the Hamilton flow for $P$ and thus equals a union of null bicharacteristics for $P$. Therefore, by (18) and (19),

$$
\text { WF } U_{j}=\text { conic-closure }\left(\Gamma_{p^{+}}^{j} \cup \Gamma_{p^{-}}^{j} \cup \Gamma_{s^{+}}^{j} \cup \Gamma_{s^{-}}^{j}\right) \text {, for } j=1,2 \text {. }
$$

The bicharacteristics $\Gamma_{p / s, \pm}$ are distinguishable (the $p$-wave is faster than the $s$-wave), and the forward and backward waves for each $j$ are distinguishable. (The "forward" wave is on the light cone $\{\tau<0\}$ and the "backward" wave is on the light cone $\{\tau>0\}$.) It follows from (16) that $\Gamma_{p^{+}}^{1}=\Gamma_{p^{+}}^{2}, \Gamma_{p^{-}}^{1}=\Gamma_{p^{-}}^{2}, \Gamma_{s^{+}}^{1}=\Gamma_{s^{+}}^{2}$, and $\Gamma_{s^{-}}^{1}=\Gamma_{s^{-}}^{2}$ outside $\Omega$ for $t \in(0, T)$.

2.5. The wave speeds $c_{p / s}$ are determined in the interior by the Dirichletto-Neumann map.

Theorem 2.4 (Rachele [R I], Theorem 4.4). Suppose that the conditions of Theorem 2.3 hold, and suppose that there are unique distance-minimizing geodesics between boundary points with respect to the metrics $\left(1 / c_{p / s}^{2}\right) d x^{i} d x^{i}$ for wave speeds $c_{p}=\sqrt{(\lambda+2 \mu) / \rho}$ and $c_{s}=\sqrt{\mu / \rho} ;$ these geodesics all exit $\Omega$ before time $T<$ $\infty$; and they do not graze $\partial \Omega$. Then $\Lambda_{\lambda_{1}, \mu_{1}, \rho_{1}}=\Lambda_{\lambda_{2}, \mu_{2}, \rho_{2}}$ on $\partial \Omega \times(0, T)$ implies

$$
\sqrt{\frac{\lambda_{1}+2 \mu_{1}}{\rho_{1}}}=\sqrt{\frac{\lambda_{2}+2 \mu_{2}}{\rho_{2}}} \text { and } \sqrt{\frac{\mu_{1}}{\rho_{1}}}=\sqrt{\frac{\mu_{2}}{\rho_{2}}} \quad \text { in } \Omega \text {. }
$$

\section{THE BOUNDARY POLARIZATION IN ISOTROPIC ELASTODYNAMICS HAS NO INFORMATION ABOUT THE DENSITY IN THE INTERIOR}

3.1. Definition of the polarization set $\mathrm{WF}_{\mathrm{pol}} U$ and polarization vectors $w$. To define the polarization set $\mathrm{WF}_{\text {pol }} U$ of a distribution $U \in \mathcal{D}^{\prime}\left(\mathbb{R}^{4}, \mathbb{R}^{4}\right)$ we consider, following Dencker $[\mathrm{De}], 1 \times 3$ systems $F$ of pseudodifferential operators of order zero. The polarization set of $U$ is the collection of $(t, x, \tau, \xi) \in T^{*}\left(\mathbb{R}^{4}\right)$ together with the polarization vectors $w(t, x, \tau, \xi)$ that make up the intersection of the kernels (that is, the orthogonal spaces) of the principal symbols $f(t, x, \tau, \xi)$ of $\operatorname{such} F$ with $F U \in C^{\infty}$. That is, we define

$$
\mathrm{WF}_{\mathrm{pol}} U=\bigcap_{F U \in C^{\infty}\left(\mathbb{R}^{3}\right)}\{(t, x, \tau, \xi ; w): w \in \operatorname{ker} f(t, x, \tau, \xi)\} .
$$

The product $F U$ is given by the usual inner product.

Dencker [De], Theorem 4.2, characterizes the polarization set (for solutions $U$ of systems of differential equations of real principal type) as a union of sections $w(t, x, \tau, \xi)$ over bicharacteristics $\Gamma \in \mathrm{WF} U$ of $P$, that solve

$$
V_{q} w=A w \quad \text { on } \Gamma \text {. }
$$


Here $V_{p}$ is the Hamilton vector field of the principal symbol $p$ of $P$ (cf. (10) and (13)); and $A=-\frac{1}{2}\{\tilde{p}, p\}-i \tilde{p} p_{m-1}^{s}$ is defined in terms of $p$,

$$
\widetilde{p}(t, x, \tau, \xi)=-\left[\left(\tau^{2}-c_{p}^{2}|\xi|^{2}\right) I+\left(c_{p}^{2}-c_{s}^{2}\right)(\xi \otimes \xi)\right],
$$

the subprincipal symbol $p_{m-1}^{s}$ of $P$, and the Poisson bracket $\{\cdot, \cdot\}$.

We denote by

$$
w_{p / s, \pm}(\alpha)=w_{p / s, \pm}(\alpha)(t, x, \tau, \xi)
$$

(with $\alpha>0$ ) the smooth section of the cotangent bundle $T^{*}\left(\mathbb{R} \times \mathbb{R}^{3}\right)$ that solves (21) on $\Gamma_{p / s, \pm}(\underline{x}, \alpha \underline{\xi})$. In particular, $w_{p / s, \pm}=w_{p / s, \pm}(1)$ denotes the polarization vector with $\alpha=1$. Given $\underline{\xi} \neq 0$ and $\underline{\xi}^{\perp}$ with $\underline{\xi}^{\perp} \cdot \underline{\xi}=0$, we denote by

$$
w_{p / s}^{\perp}(\alpha)(t, x, \tau, \xi)
$$

the polarization vector $w_{p / s}(\alpha)(t, x, \tau, \xi)$ with initial values $w_{p / s, \pm}^{\perp}(\alpha)=\underline{\xi}^{\perp}$ at $\left(0, \underline{x}, \mp c_{p / s} \alpha|\underline{\xi}|, \alpha \underline{\xi}\right)$. In Section [3.2, $w_{p / s, \pm}(\alpha)(t, x, \tau, \xi)$ denotes the polarization vector with initial values $w_{p / s, \pm}(\alpha)=\underline{\xi}$ at $\left(0, \underline{x}, \mp c_{p / s} \alpha|\underline{\xi}|, \alpha \underline{\xi}\right)$. We write $w_{p / s}=$ $w_{p / s,+}$ and $w_{p / s}^{\perp}=w_{p / s,+}^{\perp}$ for the polarization vectors over forward bicharacteristics of $P$.

3.2. Uniqueness of the polarization vectors $w_{p / s,+}$ and $w_{p / s,+}^{\perp}$ outside $\Omega$. We show in [R ], Theorem 5.1, that the $s$-wave polarization vectors $w_{s,+}^{\perp}$ are determined outside $\Omega$ by the Dirichlet-to-Neumann map:

Theorem 3.1 (Rachele [ $[\mathrm{R}]$, Theorem 5.1). For any $\underline{x} \notin \Omega$, any initial direction $\underline{\xi} \neq 0$, and any initial polarization $\underline{\xi}^{\perp} \neq 0$ with $\underline{\xi}^{\perp} \cdot \underline{\xi}=0$, let $w_{s,+}^{\perp}(t, x, \tau, \xi)$ denote the solution of (21) on $\Gamma_{s,+}(\underline{x}, \underline{\xi})$ with initial values $w_{s,+}^{\perp}=\underline{\xi}^{\perp}$ at $\left(0, \underline{x},-c_{s}(\underline{x})|\underline{\xi}|, \underline{\xi}\right)$. If $\bar{x} \notin \Omega$ with $\left(\bar{t}, \bar{x},-c_{s}(\bar{x})|\bar{\xi}|, \bar{\xi}\right) \in \Gamma_{s,+}(\underline{x}, \underline{\xi})$ for some $\bar{t}, \bar{\xi}$, then the polarization vector $w_{s,+}^{\perp}$ is determined by the Dirichlet-to-Neumann map at $\left(\bar{t}, \bar{x},-c_{s}(\bar{x})|\bar{\xi}|, \bar{\xi}\right)$.

Below we modify the proof of [R I], Theorem 5.1, to arrive at the analogous result for $p$-waves:

Theorem 3.2. For any $\underline{x} \notin \Omega$ and any initial direction $\underline{\xi} \neq 0$, let $w_{p,+}(t, x, \tau, \xi)$ denote the solution of (21) on $\Gamma_{p,+}(\underline{x}, \underline{\xi})$ with initial values $w_{p,+}=\underline{\xi}$ at $\left(0, \underline{x},-c_{p}(\underline{x})|\underline{\xi}|, \underline{\xi}\right)$. If $\bar{x} \notin \Omega$ with $\left(\bar{t}, \bar{x},-c_{p}(\bar{x})|\bar{\xi}|, \bar{\xi}\right) \in \Gamma_{p,+}(\underline{x}, \underline{\xi})$ for some $\overline{\bar{t}}, \bar{\xi}$, then the polarization vector $w_{p,+}$ is determined by the Dirichlet-to-Neumann map at $\left(\bar{t}, \bar{x},-c_{p}(\bar{x})|\bar{\xi}|, \bar{\xi}\right)$.

Proof. For $\left(\lambda_{1}, \mu_{1}, \rho_{1}\right)$ and $\left(\lambda_{2}, \mu_{2}, \rho_{2}\right)$ with $\Lambda_{\lambda_{1}, \mu_{1}, \rho_{1}}=\Lambda_{\lambda_{2}, \mu_{2}, \rho_{2}}$ we construct solutions $U_{j}$ of the Cauchy problem (3) as in the proof of [R I], Theorem 3.1, with initial data $\psi^{0}$ and $\psi^{1}$ (to be chosen later) supported outside $\Omega$. By [ $[\mathbf{R ~ I}$, Theorem 3.1, these $U_{j}$ are determined outside $\Omega$, and so their polarization sets agree outside $\Omega$ :

$$
\mathrm{WF}_{\mathrm{pol}} U_{1}=\mathrm{WF}_{\mathrm{pol}} U_{2} \quad \text { outside } \Omega .
$$

We now choose initial data $\psi_{0}$ and $\psi_{1}$, as in the proof of [R I], Theorem 5.1, again with "minimal" wave front set so that the wave front sets of the corresponding solutions $U_{1}$ and $U_{2}$ will consist of only a few null bicharacteristic strips of $P$. In fact, we choose

$$
\psi_{0}=h(x) \underline{\xi} \quad \text { and } \quad \psi_{1}=0
$$


where WF $h(x)=\{(\underline{x}, \alpha \underline{\xi}): \alpha>0\}$. (See $[\underline{\mathrm{R} \mathrm{I}}$, Remark 4.1 on the existence of such distributions $h(x)$.

We compute upper and lower bounds on the polarization set of $U_{j}$ in terms of the polarization vectors $w_{p / s, \pm}$ (cf. (23)). In fact, we show in Lemma 3.3 that the polarization set of $U_{j}$ does contain the polarization vector $w_{p,+}$, but contains at most the span of the polarization vectors $w_{p / s, \pm}(\alpha), \alpha>0$. It follows from the fact that the bicharacteristics $\Gamma_{p / s, \pm}^{j}$ are disjoint and distinguishable (for each $j$ ) and from the fact that the polarization sets $\mathrm{WF}_{\text {pol }} U_{j}$ agree outside $\Omega$ (cf. (24)) that the polarization vectors making up the polarization set of $U_{j}$ are determined outside $\Omega$. In particular, the polarization vector $w_{p,+}$ is determined. This completes the proof of the theorem.

Lemma 3.3. For the $U_{j}$ constructed in the proof of Theorem 3.2 and for polarization vectors $w_{p / s, \pm}^{j}(\alpha)$ solving (21) on $\Gamma_{p / s, \pm}^{j}$ with initial values $w_{p / s, \pm}^{j}(\alpha)=\underline{\xi}$ at $\left(0, \underline{x}, \mp c_{p / s} \alpha|\underline{\xi}|, \alpha \underline{\xi}\right)$ we have

$$
\begin{gathered}
\left\{\left(\gamma, w_{p,+}^{j}(\gamma)\right): \gamma=(t, x, \tau, \xi) \in \Gamma_{p,+}^{j}(\underline{x}, \underline{\xi})\right\} \\
\subseteq \mathrm{WF}_{\mathrm{pol}} U_{j} \\
\subseteq \bigcup_{p / s, \pm}\left\{\left(\gamma, \beta w_{p / s, \pm}^{j}(\alpha)(\gamma)\right): \alpha, \beta>0, \gamma \in \Gamma_{p / s, \pm}^{j}(\underline{x}, \alpha \underline{\xi})\right\} .
\end{gathered}
$$

Proof. To show that the upper bound of (26) holds, we describe the polarization set of $U_{j}$ by referring to Dencker's characterization (21). In particular, we observe that the polarization vectors in the polarization set $\mathrm{WF}_{\mathrm{pol}} U_{j}$ of $U_{j}$

- lie over bicharacteristics in the wave front set of $U_{j}$, and

- have initial polarization that agrees with some polarization vector of the initial data $\left.U_{j}\right|_{t=0}=\psi_{0}$.

Therefore, to describe $\mathrm{WF}_{\mathrm{pol}} U_{j}$, we describe $\mathrm{WF} U_{j}$ and $\mathrm{WF}_{\mathrm{pol}}\left(\left.U_{j}\right|_{t=0}\right)$.

We have observed in the proof of Theorem 2.3 that the wave front set of $U_{j}$ is contained in the union of the bicharacteristics $\Gamma_{p / s, \pm}^{j}(\underline{x}, \alpha \underline{\xi})$ that pass through $\left(0, \underline{x}, \mp c_{p / s}(\underline{x}) \alpha|\underline{\xi}|, \alpha \underline{\xi}\right)$, that is,

$$
\mathrm{WF} U_{j} \subseteq \bigcup_{p / s, \pm}\left\{\Gamma_{p / s, \pm}^{j}(\underline{x}, \alpha \underline{\xi}): \alpha>0\right\} .
$$

We show below in Lemma 3.4 that the polarization set of the initial data $\left.U_{j}\right|_{t=0}$ is restricted to the span of $\underline{\xi}$ over the points $(\underline{x}, \alpha \underline{\xi}), \alpha>0$. It follows that the upper bound in (26) holds.

Lemma 3.4. For the $U_{j}$ constructed in the proof of Theorem 3.2,

$$
\mathrm{WF}_{\mathrm{pol}}\left(\left.U_{j}\right|_{t=0}\right)=\{(\underline{x}, \alpha \underline{\xi} ; \beta \underline{\xi}): \alpha>0, \beta \in \mathbb{R}\} .
$$

Proof. We recall that $\left.U_{j}\right|_{t=0}=\psi_{0}=h(x) \underline{\xi}$. We first show that

$$
\mathrm{WF}_{\text {pol }} \psi_{0} \subseteq\{(\underline{x}, \alpha \underline{\xi} ; \beta \underline{\xi}): \alpha>0, \beta \in \mathbb{R}\}
$$

by considering the pseudodifferential operators $F$ with (principal) symbol $f(x, \xi)=$ $\left[I-\psi(x, \xi) \frac{\xi}{|\underline{\xi}|} \otimes \frac{\xi}{|\underline{\xi}|}\right] v(x, \xi)$, where $\psi(x, \xi)$ is any smooth function on $\mathbb{R}^{3} \times \mathbb{R}^{3}$ that is homogeneous of order 0 in $\xi$ and has values $\psi=1$ on $\{(\underline{x}, \alpha \underline{\xi}): \alpha>0\}$, and 
$v(x, \xi)$ is any smooth vector field on $T^{*} \mathbb{R}^{3}$ that is homogeneous of order 0 in $\xi$ and has values in $\mathbb{R}^{3}$.

To show that (28) holds, we will apply the definition (20) of the polarization set. We note that the intersection, over all $\psi$ and $v$, of $\{(x, \xi, w): w \in \operatorname{ker} f(x, \xi)\}$ is actually equal to the right side of (28). Then, to see that each $F \psi_{0}$ is smooth, we notice that each of the (scalar) amplitudes $f(x, \xi) \underline{\xi}$ of

$$
\begin{aligned}
h(x) & \longrightarrow\left(F\left(x, D_{x}\right) \underline{\xi}\right)(h(x)) \\
& =\int e^{i x \cdot \xi} f(x, \xi) \underline{\xi} \hat{h}(\xi) d \xi \\
& =F(h(x) \underline{\xi})=F \psi_{0}
\end{aligned}
$$

vanishes in a conic neighborhood of WF $h=\{(\underline{x}, \alpha \underline{\xi}): \alpha>0\}$.

To show that $\mathrm{WF}_{\text {pol }} \psi_{0}$ is actually given by the upper bound in (28), we observe that for any zeroth-order pseudodifferential operator $F$ on $\mathbb{R}^{3}$ with $(F \underline{\xi}) h=F \psi_{0} \in$ $C^{\infty}$ it is the case that

$$
\mathrm{WF}(h) \subseteq \mathrm{WF}((F \underline{\xi}) h) \cup\{(x, \xi): f(x, \xi) \underline{\xi}=0\},
$$

that is,

$$
\{(\underline{x}, \alpha \underline{\xi}): \alpha>0\} \subseteq\{(x, \xi): f(x, \xi) \underline{\xi}=0\},
$$

where $f(x, \xi)$ is the principal symbol of $F$. It follows that $f(\underline{x}, \alpha \underline{\xi}) \underline{\xi}=0$; that is, $\underline{\xi} \in \operatorname{ker} f(\underline{x}, \alpha \underline{\xi})$ for any $\alpha>0$.

To show that the lower bound in (26) also holds, that is, that the initial data $\psi_{0}$ does induce compressional-wave polarization of the form $w_{p,+}^{j}$ in $U_{j}$, we show that it is enough to prove that $\Gamma_{p,+}^{j} \subseteq \mathrm{WF}\left(U_{j}\right)$. In fact, by [De], Proposition 2.5, $\pi_{(t, x, \tau, \xi)}\left(\mathrm{WF}_{\mathrm{pol}} U_{j}\right) \backslash 0=\mathrm{WF} U_{j}$. It follows that if $\Gamma_{p,+}^{j} \subseteq \mathrm{WF}\left(U_{j}\right)$ holds, then some part of $\left(\mathrm{WF}_{\mathrm{pol}} U_{j}\right) \backslash 0$ lies over $\Gamma_{p,+}^{j}$. The polarization set over $\Gamma_{p,+}^{j}$ is a union of solutions of (21) that have initial polarization contained in $\mathrm{WF}_{\mathrm{pol}}\left(\left.U_{j}\right|_{t=0}\right)$. By (27) the initial values $\psi_{0}$ of $U_{j}$ have polarization only with direction $\beta \underline{\xi}, \beta \in \mathbb{R}$ (at $(\underline{x}, \alpha \underline{\xi}), \alpha>0)$. This implies that the $(p,+)$-polarization of $U_{j}$ is, in fact, the span of the polarization vectors $w_{p,+}^{j}(\alpha), \alpha>0$. Therefore, the lower bound in (26) holds.

Next, we observe that $\Gamma_{p,+}^{j} \subseteq \mathrm{WF}\left(U_{j}\right)$ holds if $(\underline{x}, \underline{\xi}) \in \mathrm{WF}\left(\left.u_{p}^{+}\right|_{t=0}\right)$, where

$$
u_{p / s}^{ \pm}=\sum_{\underline{m}} \int e^{i \varphi_{p / s}^{ \pm}(t, x, \eta)} e_{p / s, \pm}^{\cdot, \underline{m}}(0)(t, x, \eta)\left(\hat{\psi}_{0}\right)_{\underline{m}}(\eta) d \eta
$$

are terms in the sum (4) making up $U_{j}$. In fact, as in the proof of Theorem 2.3 (cf. (19) and (17)), we have WF $\left.\left(\left.u_{p}^{+}\right|_{t=0}\right) \subseteq \pi_{(x, \xi)}\left(\mathrm{WF} u_{p}^{+}\right)\right|_{t=0}$ and WF $u_{p / s}^{ \pm} \subseteq$ $\left\{\Gamma_{p / s, \pm}(\underline{x}, \alpha \underline{\xi}): \alpha>0\right\}$. Thus, if $(\underline{x}, \underline{\xi}) \in \mathrm{WF}\left(\left.u_{p}^{+}\right|_{t=0}\right)$ holds, then $\emptyset \neq \mathrm{WF}\left(u_{p}^{+}\right) \subseteq$ $\left\{\Gamma_{p,+}(\underline{x}, \alpha \underline{\xi}): \alpha>0\right\}$. Again, referring to the proof of Theorem 2.3 we note that the $\Gamma_{p / s, \pm}^{j}$ are disjoint (for each $j=1,2$ ), and so WF $U_{j}=\bigcup_{p / s, \pm} \mathrm{WF} u_{p / s}^{ \pm}$. Also, $\mathrm{WF}\left(U_{j}\right)$ is a union of bicharacteristics of $P$, and so $\mathrm{WF}\left(u_{p}^{+}\right)=\left\{\Gamma_{p,+}(\underline{x}, \alpha \underline{\xi}): \alpha>\right.$ $0\}$, and $\left\{\Gamma_{p,+}(\underline{x}, \alpha \underline{\xi}): \alpha>0\right\} \subseteq \mathrm{WF}\left(U_{j}\right)$. 
Now, to show $(\underline{x}, \underline{\xi}) \in \mathrm{WF}\left(\left.u_{p}^{+}\right|_{t=0}\right)$ we observe that

$$
\begin{aligned}
\left.u_{p}^{+}\right|_{t=0} & =\left.\sum_{\underline{m}} \int e^{i \varphi_{p}^{+}(t, x, \eta)} e_{p,+}^{\cdot, \underline{m}}(0)(t, x, \eta)\left(\widehat{\psi_{0}}\right)_{\underline{m}}(\eta) d \eta\right|_{t=0} \\
& =\sum_{\underline{m}} \int e^{i\left[\varphi_{p}^{+}(0, x, \eta)-y \cdot \eta\right]} e_{p, \underline{m}}^{\cdot,+}(0)(0, x, \eta)\left(\psi_{0}\right)_{\underline{m}}(y) d y d \eta \\
& =\int e^{i\left[\varphi_{p}^{+}(0, x, \eta)-y \cdot \eta\right]} e_{p,+}(0)(0, x, \eta) \underline{\xi} h(y) d y d \eta \\
& =\int e^{i x \cdot \eta} e_{p,+}(0)(0, x, \eta) \underline{\xi} \hat{h}(\eta) d \eta,
\end{aligned}
$$

with the last equality holding due to the initial values $\varphi_{p / s}^{ \pm}(0, x, \eta)=x \cdot \eta$ of $\varphi_{p}^{+}$ given in (7). By (12) $\left.u_{p}^{+}\right|_{t=0}=\frac{1}{2} \mathrm{P}_{\eta}\left(x, D_{x}\right) \underline{\xi}(h)$, where $\mathrm{P}_{\eta}\left(x, D_{x}\right) \underline{\xi}(h)=\int e^{i x \cdot \eta}\left[\frac{\eta}{|\eta|}\right.$ $\left.\otimes \frac{\eta}{\mid \eta}\right] \underline{\xi} \hat{h}(\eta) d \eta$ is the $1 \times 3$ system of pseudodifferential operators with symbol $\mathrm{P}_{\eta}(x, \eta) \underline{\xi}=\left(\frac{\eta}{|\eta|} \otimes \frac{\eta}{|\eta|}\right) \underline{\xi}$ (acting on the scalar $\left.h\right)$.

For each component $(l=1,2,3)$ of the amplitude $\mathrm{P}_{\eta}(x, \eta) \underline{\xi}$, we have

$$
\mathrm{WF} h \subseteq \mathrm{WF}\left(\left(\mathrm{P}_{\eta}\left(x, D_{x}\right) \underline{\xi}\right)_{l} h\right) \cup\left\{(x, \eta):\left(\mathrm{P}_{\eta}(x, \eta) \underline{\xi}\right)_{l}=0\right\} .
$$

The wave front set of $h$ is the ray through $(\underline{x}, \underline{\xi})$, and $\left(\mathrm{P}_{\eta}(\underline{x}, \underline{\xi}) \underline{\xi}\right)_{l}=\underline{\xi}_{l} \neq 0$ for some $l$. Therefore, $(\underline{x}, \underline{\xi})$ is, in fact, in $\mathrm{WF}\left(\mathrm{P}_{\eta}\left(x, D_{x}\right) \underline{\xi}(h)\right)=\mathrm{WF}\left(\left.u_{p}^{+}\right|_{t=0}\right)$.

This completes the proof of the lower bound in (26)).

3.3. Conclusion: Boundary polarization contains no information about the density in the interior. We first compute the terms involved in the ODE (21) which describes the propagation of the polarization vectors $w_{p / s}^{+}$. To shorten the calculation of $A$ we follow Dencker [De, Example 4.3. We define $\widetilde{P}$ to be the pseudodifferential operator with (principal) symbol $\tilde{p}$ given in (22). Then the pseudodifferential operator $Q=\tilde{P} P$ has principal symbol $q I$ with $q$ scalar and of real principal type. That is, by (10) and (22),

$$
q(t, x, \tau, \xi)=\tilde{p} p=\rho\left(\tau^{2}-c_{s}^{2}|\xi|^{2}\right)\left(\tau^{2}-c_{p}^{2}|\xi|^{2}\right) .
$$

For $w \in \operatorname{ker} p(t, x, \tau, \xi)$ we have $A=A_{q}$ where $A_{q}=-i q^{s}$ with $q^{s}$ the subprincipal symbol of $Q$. That is, for $Q_{j}=Q\left(\lambda_{j}, \mu_{j}, \rho_{j}\right)$, the polarization vectors of $U_{j}$ solve

$$
V_{q} w=A_{q} w,
$$

where $V_{q}$ is the Hamilton vector field of $q$ (cf. (13)). In fact, writing $c_{\lambda}^{2}=\lambda / \rho$, we have

$\left.V_{q}\right|_{\tau=\mp c_{p / s}|\xi|}=\mp(+/-) 2 \rho c_{p / s}^{2}\left(c_{p}^{2}-c_{s}^{2}\right)|\xi|^{3} \cdot\left[\frac{\partial_{t}}{c_{p / s}} \pm \frac{\xi}{|\xi|} \cdot \nabla_{x} \mp\left(\log c_{p / s}\right)_{x}|\xi| \cdot \nabla_{\xi}\right]$,

$p_{1}(t, x, \tau, \xi)$ consists of the lower-order terms of the symbol of $P$ and is given by

$$
\begin{aligned}
p_{1}(t, x, \tau, \xi) & =-i\left[\lambda_{x} \otimes \xi+\left(\mu_{x} \cdot \xi\right) I+\xi \otimes \mu_{x}\right] \\
& =-i \rho\left[c_{\lambda}^{2}\left(\log \rho c_{\lambda}^{2}\right)_{x} \otimes \xi+\left[c_{s}^{2}\left(\log \rho c_{s}^{2}\right)_{x} \cdot \xi\right] I+c_{s}^{2} \xi \otimes\left(\log \rho c_{s}^{2}\right)_{x}\right],
\end{aligned}
$$


where

In addition,

$$
u(\forall) \frac{1}{2}[u \otimes v+v \otimes u] .
$$

$$
\begin{aligned}
& A_{q}=-i q^{s}=-i \widetilde{p} p_{1}-\sum_{j}\left(\partial_{\xi_{j}} \widetilde{p}\right)\left(\partial_{x_{j}} p\right)+\frac{1}{2}\left(\sum_{j} \partial_{x_{j}} \partial_{\xi_{j}} q\right) I \\
&= \rho \sum_{j}\left[2 c_{p}^{2} \xi_{j} I-2\left(c_{p}^{2}-c_{s}^{2}\right)\left(\xi\left(s e_{j}\right)\right] \cdot\left[-\left(c_{p}^{2}-c_{s}^{2}\right)_{x_{j}}(\xi \otimes \xi)\right.\right. \\
&\left.+(\log \rho)_{x_{j}}\left(\left(\tau^{2}-c_{s}^{2}|\xi|^{2}\right) I-\left(c_{p}^{2}-c_{s}^{2}\right)(\xi \otimes \xi)\right)-\left(c_{s}^{2}\right)_{x_{j}}|\xi|^{2} I\right] \\
&+\rho\left[\left(\tau^{2}-c_{p}^{2}|\xi|^{2}\right) I+\left(c_{p}^{2}-c_{s}^{2}\right)(\xi \otimes \xi)\right] \\
& \cdot\left(\left(c_{p}^{2}-2 c_{s}^{2}\right)\left[\log \rho\left(c_{p}^{2}-2 c_{s}^{2}\right)\right]_{x} \otimes \xi\right. \\
&\left.+c_{s}^{2}\left[\left(\log \rho c_{s}^{2}\right)_{x} \cdot \xi\right] I+c_{s}^{2} \xi \otimes\left(\log \rho c_{s}^{2}\right)_{x}\right) \\
&-\rho \sum_{j}\left((\log \rho)_{x_{j}}\left[c_{p}^{2}\left(\tau^{2}-c_{s}^{2}|\xi|^{2}\right) \xi_{j}+c_{s}^{2}\left(\tau^{2}-c_{p}^{2}|\xi|^{2}\right) \xi_{j}\right]\right. \\
&+\left(c_{p}^{2}\right)_{x_{j}}\left(\tau^{2}-c_{s}^{2}|\xi|^{2}\right) \xi_{j}+\left(c_{s}^{2}\right)_{x_{j}}\left(\tau^{2}-c_{p}^{2}|\xi|^{2}\right) \xi_{j} \\
&\left.-c_{p}^{2}|\xi|^{2}\left(c_{s}^{2}\right)_{x_{j}} \xi_{j}-c_{s}^{2}|\xi|^{2}\left(c_{p}^{2}\right)_{x_{j}} \xi_{j}\right) I
\end{aligned}
$$

for

$$
\begin{aligned}
& \gamma_{1}=\left[\xi \cdot(\log \rho)_{x}\right] c_{p}^{2}\left(c_{p}^{2}-c_{s}^{2}\right)|\xi|^{2} \delta_{\tau^{2}=c_{p}^{2}|\xi|^{2}}-\left[\xi \cdot\left(c_{s}^{2}\right)_{x}\right] c_{p}^{2}|\xi|^{2} \\
& +\left[\xi \cdot\left(c_{p}^{2}\right)_{x}\right]\left[\left(c_{p}^{2}-c_{s}^{2}\right)|\xi|^{2} \delta_{\tau^{2}=c_{p}^{2}|\xi|^{2}}-c_{s}^{2}|\xi|^{2}\right], \\
& \gamma_{2}=\left[\xi \cdot(\log \rho)_{x}\right]\left[-2 c_{s}^{2}\left(c_{p}^{2}-c_{s}^{2}\right)\right]-\left[\xi \cdot\left(c_{p}^{2}-c_{s}^{2}\right)_{x}\right]\left[2 c_{s}^{2}\right], \\
& v_{1}=-(\log \rho)_{x}\left(c_{p}^{2}-c_{s}^{2}\right)|\xi|^{2}\left[\left(c_{p}^{2}-2 c_{s}^{2}\right) \delta_{\tau^{2}=c_{p}^{2}|\xi|^{2}}\right] \\
& +\left(c_{s}^{2}\right)_{x}\left(c_{p}^{2}-c_{s}^{2}\right)|\xi|^{2}\left[2-\delta_{\tau^{2}=c_{s}^{2}|\xi|^{2}}\right], \\
& v_{2}=(\log \rho)_{x}\left(c_{p}^{2}-c_{s}^{2}\right)|\xi|^{2}\left[c_{s}^{2} \delta_{\tau^{2}=c_{s}^{2}|\xi|^{2}}\right]+2\left(c_{s}^{2}\right)_{x}\left(c_{p}^{2}-c_{s}^{2}\right)|\xi|^{2} \delta_{\tau^{2}=c_{s}^{2}|\xi|^{2}} \\
& +\left(c_{p}^{2}\right)_{x}\left(c_{p}^{2}-c_{s}^{2}\right)|\xi|^{2} \delta_{\tau^{2}=c_{p}^{2}|\xi|^{2}} .
\end{aligned}
$$

We denote by ' and by $\frac{d}{d s}$ the derivative with respect to arclength in the direction of the bicharacteristic $\Gamma_{p / s}^{ \pm}(\underline{x}, \underline{\xi}) \quad$ (cf. (14)) ; that is,

$$
\frac{d}{d s}=\frac{\partial_{t}}{c_{p / s}} \pm \frac{\xi}{|\xi|} \cdot \nabla_{x} \mp\left(\log c_{p / s}\right)_{x}|\xi| \cdot \nabla_{\xi} .
$$

It follows that the ODE (29) reduces to

$$
\left(w_{p / s}^{ \pm}\right)^{\prime}=\frac{d w_{p / s}}{d s}=\frac{(+/-)\left[\gamma_{1} I+\gamma_{2}(\xi \otimes \xi)+\xi \otimes v_{1}+v_{2} \otimes \xi\right]}{2 c_{p / s}^{2}\left(c_{p}^{2}-c_{s}^{2}\right)|\xi|^{3}} w_{p / s}^{ \pm} .
$$

In the case of a forward $p$-wave (i.e., $\tau=-c_{p}|\xi|$ ) we have $w_{p}=\alpha N$ where $N=\frac{\xi}{|\xi|}=\frac{d x}{d s}$. Therefore, the ODE in this case reduces to

$$
\alpha^{\prime} N+\alpha N^{\prime}=\left.\frac{d w_{p}}{d s}\right|_{\tau=-c_{p}|\xi|}=\alpha \frac{\left[\gamma_{1}+\gamma_{2}|\xi|^{2}+v_{1} \cdot \xi\right] N+v_{2}|\xi|}{2 c_{p}^{2}\left(c_{p}^{2}-c_{s}^{2}\right)|\xi|^{3}}
$$


that is, given (31),

$$
\alpha^{\prime}=\alpha G\left(c_{p}, c_{s}\right)
$$

where $G\left(c_{p}, c_{s}\right)$ does not depend on $\rho$. (Note that by (14),

$$
N^{\prime}=\left(\frac{\xi}{|\xi|}\right)^{\prime}=\left(\log c_{p}\right)_{x}+\left(\log c_{p}\right)^{\prime} N
$$

while $\left.v_{2}|\xi| /\left(2 c_{p}^{2}\left(c_{p}^{2}-c_{s}^{2}\right)|\xi|^{3}\right)=\left(\log c_{p}\right)_{x}\right)$. It follows that the $p$-wave boundary polarization does not contain any information about the value of the density $\rho$ in the interior.

In the case of a forward $s$-wave (i.e., $\tau=-c_{s}|\xi|$ ) we have $w_{s} \perp \xi$; so the ODE reduces to

$$
\left.\frac{d w_{s}(t, x, \tau, \xi)}{d s}\right|_{\tau=-c_{s}|\xi|}=\frac{-\left[\gamma_{1} w_{s}+\left(v_{1} \cdot w_{s}\right) \xi\right]}{2 c_{s}^{2}\left(c_{p}^{2}-c_{s}^{2}\right)|\xi|^{3}} .
$$

It follows that

$$
\frac{d}{d s}\left(\log \left|w_{s}\right|^{2}\right)=\frac{2 w_{s}}{\left|w_{s}\right|^{2}} \cdot \frac{d w_{s}}{d s}=\frac{-\gamma_{1}}{c_{s}^{2}\left(c_{p}^{2}-c_{s}^{2}\right)|\xi|^{3}}
$$

and

$$
\frac{d}{d s}\left(w_{s} \cdot \frac{w_{s}(0)}{\left|w_{s}(0)\right|}\right)=\left[\operatorname{proj}_{w_{s}^{\perp}} w_{s}^{\prime}\right] \cdot \frac{w_{s}(0)}{\left|w_{s}(0)\right|}
$$

where $w_{s}(0)$ is the polarization vector at the boundary.

Each of these ODEs, (33) and (34), has right-hand sides that do not depend on $\rho(x)$ with $x \in \Omega$, when $\tau=-c_{s}|\xi|$. It follows that $\left|w_{s}\right|$ and $\operatorname{Arccos}\left(\frac{w_{s}}{\left|w_{s}\right|} \cdot \frac{w_{s}(0)}{\left|w_{s}(0)\right|}\right)$ do not depend on $\rho$ in the interior. Therefore, the $s$-wave boundary polarization also contains no information about the value of the density $\rho$ in the interior.

\section{UNIQUENESS OF THE DENSITY}

4.1. Using the transport equations to describe the propagation of "lowerorder polarization". Here we return to the representation of the elastic displacement wave in terms of solutions of the Cauchy problem (3), in particular, in terms of the Fourier integral operators described in Section 2.2. The amplitudes of these Fourier integral operators solve algebraic equations (9) and satisfy compatibility conditions (11).

We reduce the compatibility conditions (11) (for top-order and lower-order terms of the $p$-wave FIO amplitudes) to the transport equations (37) and (39), ODEs along the bicharacteristics of $P$. Writing $\alpha_{0}$ for $\left(\alpha^{\underline{m}}\right)_{0}(t, x, \eta)$, we have by (18), (10), (30), 
for forward $p$-waves (i.e., $\left.\tau=-c_{p}|\xi|\right)$ :

$$
\begin{aligned}
\partial_{\tau} p & =-2 \rho \tau I \\
\partial_{\xi_{j}} p & =2 \rho\left[c_{s}^{2} \xi_{j} I+\left(c_{p}^{2}-c_{s}^{2}\right)\left(e_{j} S \xi\right)\right], \\
\partial_{\xi_{i}} \partial_{\xi_{j}} p & =2 \rho\left[c_{s}^{2} \delta_{i j} I+\left(c_{p}^{2}-c_{s}^{2}\right)\left(e_{i} S e_{j}\right)\right], \\
i N \partial_{\tau, \xi} p \cdot \partial_{t, x} & {\left[\alpha_{0} N\right] } \\
& =i \rho\left[2 c_{p}^{2}|\xi|\left(\frac{\partial_{t}}{c_{p}}+\frac{\xi}{|\xi|} \cdot \nabla_{x}\right) \alpha_{0}+\left(c_{p}^{2}-c_{s}^{2}\right)|\xi|\left(\nabla_{x} \cdot N\right) \alpha_{0}\right], \\
-N p_{1}\left[\alpha_{0} N\right] & =i\left(\left[\xi \cdot \lambda_{x}\right]+2\left[\xi \cdot \mu_{x}\right]\right) \alpha_{0}=i \rho c_{p}^{2}\left[\xi \cdot\left(\log \rho c_{p}^{2}\right)_{x}\right] \alpha_{0}, \\
N \cdot(i / 2) \sum_{|\alpha|=2} & \sum_{l=1}^{3}\left(\partial_{\tau, \xi}^{\alpha} p\right) \cdot\left(\partial_{t, x}^{\alpha} \varphi_{p}\right)\left[\alpha_{0} N\right] \\
& =i \rho\left(-c_{p}^{2}\left(\log c_{p}\right)^{\prime}|\xi|+c_{s}^{2}\left(\nabla_{x} \cdot \xi\right)-c_{s}^{2}\left[N\left(\nabla_{x} \otimes \xi\right) N\right]\right) \alpha_{0}
\end{aligned}
$$

due to the fact that

$$
\begin{gathered}
2 N \cdot N_{t}=\partial_{t}(N \cdot N)=\partial_{t}(1)=0, \\
\left(\nabla_{x} \otimes N\right) N=\frac{1}{2}\left[\nabla_{x}(N \cdot N)\right]=\frac{1}{2}\left[\nabla_{x}(1)\right]=0, \\
\partial_{t}^{2} \varphi_{p}(t, x, \eta) \stackrel{(32)}{=}\left[c_{p} \frac{d}{d s}-c_{p} N \cdot \nabla_{x}\right] \tau=c_{p}^{2}\left[\left(\log c_{p}\right)^{\prime}|\xi|+N\left(\nabla_{x} \otimes \xi\right) N\right], \\
\nabla_{x} \cdot N=\frac{\nabla_{x} \cdot \xi}{|\xi|}-\frac{N\left(\nabla_{x} \otimes \xi\right) N}{|\xi|} .
\end{gathered}
$$

Given (8), it follows from the compatibility condition (11) for $J=0, N B_{p}\left(e_{\vec{p}}^{\cdot} \underline{\underline{m}}(\underline{k})\right)_{0}$ $=0$, that

$$
\alpha_{0}^{\prime}=-\left[\left(\log \sqrt{\rho c_{p}}\right)^{\prime}+\frac{1}{2}\left(\nabla_{x} \cdot N\right)\right] \alpha_{0} .
$$

Therefore, for $\underline{x}=x(\underline{s}) \notin \Omega, \underline{t}=t(\underline{s}), \xi(s)=\nabla_{x} \varphi_{p}(t(s), x(s), \eta)$, and $\alpha_{0}=$ $\alpha_{0}(t(s), x(s), \eta)$

$$
\alpha_{0}=\alpha_{0}(\underline{t}, \underline{x}, \eta) \frac{\sqrt{\left(\rho c_{p}\right)(\underline{t}, \underline{x}, \eta)}}{\sqrt{\rho c_{p}(x)}} \exp \left(-\frac{1}{2} \int_{\underline{s}}^{s} \nabla_{x} \cdot N d s\right)=\frac{H}{\sqrt{\rho}},
$$

where $H=H(t(s), x(s), \eta)$ depends on $c_{p}$, but not $\rho(x)$ for $x \in \Omega$.

To derive the transport equation for $\alpha_{-1}=\left(\alpha^{\underline{m}}\right)_{-1}(t, x, \eta)$ from the compatibility condition (11) for $J=-1, N\left[B_{p}\left(e_{p}\right)_{-1}+C_{p}\left(e_{p}\right)_{0}\right]=0$, we first observe, as in (35) and the calculations that follow, that

$$
N B_{p}\left(\alpha_{-1} N\right)=2 i \rho c_{p}^{2}|\xi|\left(\alpha_{-1}^{\prime}+\left[\left(\log \sqrt{\rho c_{p}}\right)^{\prime}+\frac{1}{2}\left(\nabla_{x} \cdot N\right)\right] \alpha_{-1}\right) .
$$

It follows that this compatibility condition reduces to the transport equation

$$
\alpha_{-1}^{\prime}+\left[\left(\log \sqrt{\rho c_{p}}\right)^{\prime}+\frac{1}{2}\left(\nabla_{x} \cdot N\right)\right] \alpha_{-1}=G,
$$

where

$$
G=\frac{-1}{2 i \rho c_{p}^{2}|\xi|}\left[N B_{p} h_{-1}+N C_{p}\left(\alpha_{0} N\right)\right] .
$$


We solve the transport equation (39):

$$
g \alpha_{-1}=\int g G+C
$$

where $C$ is a constant, the integration is over $p$-wave bicharacteristic segments (cf. Section [2.3), and

$$
g=\frac{\sqrt{\rho}}{H}=\frac{\sqrt{\rho c_{p}(x)}}{\alpha_{0}(\underline{t}, \underline{x}, \eta) \sqrt{\left(\rho c_{p}\right)(\underline{t}, \underline{x}, \eta)}} \exp \left(\frac{1}{2} \int_{\underline{s}}^{s} \nabla_{x} \cdot N d s\right)
$$

has $g^{\prime}=\left[\left(\log \sqrt{\rho c_{p}}\right)^{\prime}+\frac{1}{2}\left(\nabla_{x} \cdot N\right)\right] g$.

We compute $G$, given (10), (35), (36), and (38):

$$
\begin{aligned}
& N C_{p}\left(\alpha_{0} N\right) \\
& =\rho\left(2\left(c_{p}^{2}-2 c_{s}^{2}\right)\left[\log \sqrt{\rho\left(c_{p}^{2}-2 c_{s}^{2}\right)}\right]^{\prime}\left[\nabla_{x} \cdot\left(\frac{H}{\sqrt{\rho}} N\right)\right]\right. \\
& +2 c_{s}^{2}\left(\log \sqrt{\rho} c_{s}\right)_{x}\left[\nabla_{x} \otimes\left(\frac{H}{\sqrt{\rho}} N\right)\right] N-\partial_{t}^{2}\left(\frac{H}{\sqrt{\rho}} N\right) \cdot N \\
& +2 c_{s}^{2} N\left[\nabla_{x} \otimes\left(\frac{H}{\sqrt{\rho}} N\right)\right]\left(\log \sqrt{\rho} c_{s}\right)_{x}+c_{s}^{2} \Delta\left(\frac{H}{\sqrt{\rho}} N\right) \cdot N \\
& \left.+\left(c_{p}^{2}-c_{s}^{2}\right) N\left[\nabla_{x} \otimes \nabla_{x}\right] \cdot\left(\frac{H}{\sqrt{\rho}} N\right)\right) \\
& =\quad-H \sqrt{\rho}\left(c_{s}^{2}(\Delta \log \sqrt{\rho})+\left(c_{p}^{2}-c_{s}^{2}\right)\left[N(\log \sqrt{\rho})_{x x} N\right]\right. \\
& \quad+c_{s}^{2}\left|(\log \sqrt{\rho})_{x}\right|^{2}+\left(c_{p}^{2}-c_{s}^{2}\right)\left[(\log \sqrt{\rho})^{\prime}\right]^{2} \\
& +(\log \sqrt{\rho})^{\prime}\left[2\left(c_{p}^{2}-c_{s}^{2}\right)\left(\log \sqrt{c_{p}^{2}-c_{s}^{2}}\right)^{\prime}-\left(c_{p}^{2}-3 c_{s}^{2}\right)\left(\nabla_{x} \cdot N\right)\right] \\
& \quad+(\log \sqrt{\rho})_{x} \cdot\left[\left(c_{p}^{2}-3 c_{s}^{2}\right)\left(\nabla_{x} \otimes N\right)^{t} N+2 c_{s}^{2}\left(\log c_{s}\right)_{x}\right] \\
& + \text { terms that do not depend on } \rho)
\end{aligned}
$$

since, by (30) and (38),

$$
\begin{aligned}
& \partial_{\xi_{j}}\left(i p_{1}\right)=\rho\left(2\left(c_{p}^{2}-2 c_{s}^{2}\right)\left[\left(\log \sqrt{\rho\left(c_{p}^{2}-2 c_{s}^{2}\right)}\right)_{x} \otimes e_{j}\right]\right. \\
&\left.+2 c_{s}^{2}\left(\log \sqrt{\rho} c_{s}\right)_{x_{j}} I+2 c_{s}^{2}\left[e_{j} \otimes\left(\log \sqrt{\rho} c_{s}\right)_{x}\right]\right), \\
& \sqrt{\rho}\left(\alpha_{0}\right)_{x}=H\left[-(\log \sqrt{\rho})_{x}+(\log H)_{x}\right], \\
& \sqrt{\rho}\left(\alpha_{0}\right)_{x x}=H[(\log \sqrt{\rho})_{x} \otimes(\log \sqrt{\rho})_{x}-(\log \sqrt{\rho})_{x x}+(\log H)_{x x} \\
&\left.-2(\log \sqrt{\rho})_{x}(s)(\log H)_{x}+(\log H)_{x} \otimes(\log H)_{x}\right] .
\end{aligned}
$$

We note, by (32) and (37), that $\alpha_{0}=\alpha_{0}(t, x, \eta)$ solves

$$
\left[\frac{\partial_{t}}{c_{p}}+\frac{\xi}{|\xi|} \cdot \nabla_{x}\right] \alpha_{0}=\alpha_{0}^{\prime}=-\left[\left(\log \sqrt{\rho c_{p}}\right)^{\prime}+\frac{1}{2}\left(\nabla_{x} \cdot N\right)\right] \alpha_{0}
$$


so, by the definition of $H$ in (38),

$$
\partial_{t} \log H=-c_{p}\left[\left(\log \sqrt{c_{p}}\right)^{\prime}+\frac{1}{2}\left(\nabla_{x} \cdot N\right)+\left[N \cdot(\log H)_{x}\right]\right] .
$$

It follows, given the algebraic equations (9) with $J=0$, given that $N$ is in $\operatorname{ker} p\left(t, x, \partial_{t, x} \varphi_{p}\right)(\operatorname{cf}$. (8) $)$, and given that $h_{-1}$ is orthogonal to $\xi$ (cf. (8) ), that

$$
\begin{aligned}
h_{-1}= & \frac{-1}{\rho\left(c_{p}^{2}-c_{s}^{2}\right)|\xi|^{2}} B_{p}\left(\alpha_{0} N\right) \\
= & \frac{-1}{\rho\left(c_{p}^{2}-c_{s}^{2}\right)|\xi|^{2}} \cdot i \rho|\xi|\left(2 c_{p} \partial_{t}\left(\alpha_{0} N\right)+2 c_{s}^{2}\left[\nabla_{x} \otimes\left(\alpha_{0} N\right)\right]^{t} N\right. \\
& +\left(c_{p}^{2}-c_{s}^{2}\right)\left[\left[\nabla_{x} \otimes\left(\alpha_{0} N\right)\right] N+\left[\nabla_{x} \cdot\left(\alpha_{0} N\right)\right] N\right] \\
& +\left[2\left(c_{p}^{2}-2 c_{s}^{2}\right)\left(\log \sqrt{\rho\left(c_{p}^{2}-2 c_{s}^{2}\right)}\right)_{x}+4 c_{s}^{2}\left(\log \sqrt{\rho} c_{s}\right)^{\prime} N\right. \\
& \left.\left.-\frac{\partial_{t}^{2} \varphi_{p}}{|\xi|} N+c_{s}^{2} \frac{\left(\Delta \varphi_{p}\right)}{|\xi|} N+\left(c_{p}^{2}-c_{s}^{2}\right) \frac{\left[\nabla_{x} \otimes \xi\right]}{|\xi|} N\right] \alpha_{0}\right) \\
= & \frac{i H}{\left(c_{p}^{2}-c_{s}^{2}\right)|\xi| \sqrt{\rho}}(V+W),
\end{aligned}
$$

especially due to (10), (14), (30), (35), (36), (38), (44), (45), and

$$
\begin{aligned}
\partial_{t} N(t, x, \eta) & =c_{p}\left[\left(\frac{\xi}{|\xi|}\right)^{\prime}-\left(\nabla_{x} \otimes N\right)^{t} N\right] \\
& =-c_{p}\left[\left(\nabla_{x} \otimes N\right)^{t} N+\left(\log c_{p}\right)_{x}-\left(\log c_{p}\right)^{\prime} N\right] \\
\left(\nabla_{x} \otimes N\right)^{t} N & =\frac{\left(\nabla_{x} \otimes \xi\right)}{|\xi|} N-\left[N^{t} \frac{\left(\nabla_{x} \otimes \xi\right)}{|\xi|} N\right] N .
\end{aligned}
$$

Here we use the notation

$$
\begin{aligned}
& V=2 N @\left[\left(c_{p}^{2}-3 c_{s}^{2}\right)(\log \sqrt{\rho})_{x}\right] N, \\
& W=2 N @\left[-4 c_{s}^{2}\left(\log c_{s}\right)_{x}+\left(c_{p}^{2}-c_{s}^{2}\right)(\log H)_{x}\right] N \\
& +\left(c_{p}^{2}-c_{s}^{2}\right)\left(\nabla_{x} \otimes N\right)^{t} N, \\
& {[u @ v] w=\frac{1}{2}([u \otimes v] w-[v \otimes u] w)=\frac{1}{2}(u[v \cdot w]-v[u \cdot w]) .}
\end{aligned}
$$

Therefore, given that $N \cdot h_{-1}=0$, we have

$$
\begin{aligned}
N B_{p}=i \rho|\xi| & \left(-2 c_{p}\left(\partial_{t} N\right) \cdot h_{-1}+\left(c_{p}^{2}+c_{s}^{2}\right) N^{t}\left[\nabla_{x} \otimes h_{-1}\right] N\right. \\
+\left(c_{p}^{2}-c_{s}^{2}\right)\left(\nabla_{x} \cdot h_{-1}\right)+ & 2 c_{s}^{2}\left[\left(\log \sqrt{\rho} c_{s}\right)_{x} \cdot h_{-1}\right] \\
& \left.+\left(c_{p}^{2}-c_{s}^{2}\right) N^{t} \frac{\left[\nabla_{x} \otimes \xi\right]}{|\xi|} h_{-1}\right)
\end{aligned}
$$




$$
\begin{gathered}
=i \rho|\xi| \cdot \frac{i H}{\left(c_{p}^{2}-c_{s}^{2}\right)|\xi| \sqrt{\rho}}\left(-\left(c_{p}^{2}+c_{s}^{2}\right)(\log \sqrt{\rho})^{\prime}(W \cdot N)\right. \\
-\left(c_{p}^{2}-3 c_{s}^{2}\right)\left[(\log \sqrt{\rho})_{x} \cdot(V+W)\right]+\left(c_{p}^{2}-c_{s}^{2}\right)\left(\nabla_{x} \cdot V\right) \\
+\left(c_{p}^{2}+c_{s}^{2}\right)\left[N^{t}\left(\nabla_{x} \otimes V\right) N\right]+2 c_{p}^{2}\left[N^{t}\left(\nabla_{x} \otimes N\right) V\right] \\
+\left(c_{p}^{2}-c_{s}^{2}\right)\left[(\log H)_{x} \cdot V\right]+4 c_{s}^{2}\left[\left(\log c_{s}\right)_{x} \cdot V\right] \\
+ \text { terms that do not depend on } \rho) \\
=\frac{-H \sqrt{\rho}}{\left(c_{p}^{2}-c_{s}^{2}\right)} \cdot\left[\left(\left|(\log \sqrt{\rho})_{x}\right|^{2}-\left[(\log \sqrt{\rho})^{\prime}\right]^{2}\right)\left(c_{p}^{2}-3 c_{s}^{2}\right)^{2}\right. \\
+\left(\left[N^{t}(\log \sqrt{\rho})_{x x} N\right]-\Delta(\log \sqrt{\rho})\right)\left(c_{p}^{2}-c_{s}^{2}\right)\left(c_{p}^{2}-3 c_{s}^{2}\right) \\
+(\log \sqrt{\rho})^{\prime}\left(\left(c_{p}^{2}-c_{s}^{2}\right)\left(c_{p}^{2}-3 c_{s}^{2}\right)\left(\nabla_{x} \cdot N\right)\right. \\
\left.+\left(c_{p}^{2}-c_{s}^{2}\right)\left(c_{p}^{2}+c_{s}^{2}\right)^{\prime}-8 c_{s}^{2}\left(c_{s}^{2}\right)^{\prime}\right) \\
-(\log \sqrt{\rho})_{x} \cdot\left(\left(c_{p}^{2}-c_{s}^{2}\right)\left(c_{p}^{2}-3 c_{s}^{2}\right)\left(\nabla_{x} \otimes N\right)^{t} N\right. \\
\left.+\left(c_{p}^{2}-c_{s}^{2}\right)\left(c_{p}^{2}+c_{s}^{2}\right)_{x}-8 c_{s}^{2}\left(c_{s}^{2}\right)_{x}\right) \\
+\operatorname{terms} \text { that do not depend on } \rho] .
\end{gathered}
$$

It follows from (40), (41), (42), (43), and (46) that

$$
\begin{aligned}
g \alpha_{-1} & =\int g G+C \\
& =\int \frac{\sqrt{\rho}}{H} \cdot \frac{-1}{2 i \rho c_{p}^{2}|\xi|} \cdot(-H \sqrt{\rho})\left[N^{t} \cdot A(x) \cdot N\right]+C \\
& =\frac{-1}{2 i \tau} \int\left[N^{t} \cdot \frac{A(x)}{c_{p}} \cdot N\right]+C,
\end{aligned}
$$

where the rank-2 tensor $A(x)$ depends only on $x$ and is given by

$$
\begin{aligned}
& A(x)=(\log \sqrt{\rho})_{x x}\left[2\left(c_{p}^{2}-2 c_{s}^{2}\right)\right] \\
&+\left[(\log \sqrt{\rho})_{x} \otimes(\log \sqrt{\rho})_{x}\right]\left[4 c_{s}^{2} \frac{\left(c_{p}^{2}-2 c_{s}^{2}\right)}{\left(c_{p}^{2}-c_{s}^{2}\right)}\right] \\
&+(\log \sqrt{\rho})_{x} \otimes\left[2\left(c_{p}^{2}\right)_{x}-\frac{8 c_{s}^{2}}{c_{p}^{2}-c_{s}^{2}}\left(c_{s}^{2}\right)_{x}\right] \\
&-I\left[\Delta(\log \sqrt{\rho})\left(c_{p}^{2}-4 c_{s}^{2}\right)\right. \\
&-\left|(\log \sqrt{\rho})_{x}\right|^{2}\left(\left(c_{p}^{2}-4 c_{s}^{2}\right)+\frac{4 c_{s}^{4}}{c_{p}^{2}-c_{s}^{2}}\right) \\
&\left.-(\log \sqrt{\rho})_{x} \cdot\left(-\left(c_{p}^{2}\right)_{x}+\frac{8 c_{s}^{2}}{c_{p}^{2}-c_{s}^{2}}\left(c_{s}^{2}\right)_{x}\right)\right] .
\end{aligned}
$$

It follows from the fact that $N=\nabla_{x} \varphi_{p} /\left|\nabla_{x} \varphi_{p}\right|$ is the unit tangent vector to the spatial component $x(s)$ of the bicharacteristics (cf. Section [2.3), and from the fact 
that the spatial components $x(s)$ are geodesics with respect to the metric $\left(1 / c_{p}^{2}\right) e$, that these line integrals (47), when taken over all $p$-wave bicharacteristics (cf. (14)) (that is, for all $\underline{x} \notin \Omega, \underline{\xi} \in \mathbb{R}^{3} \backslash 0$, cf. (15)) make up a ray transform of the symmetric tensor field $A^{-}$(cf. (51) and Theorem 4.2) over the family of geodesics with respect to $\left(1 / c_{p}^{2}\right) e$.

The Dirichlet-to-Neumann map determines the value of the ray transform (47) over geodesic segments between boundary points of $\Omega$. (In fact, the Dirichlet-toNeumann map determines the wave displacement $U_{j}$ outside $\Omega$ (cf. Theorem 2.2) and thus it determines $g \alpha_{-1}$ at $\partial \Omega$.) We conclude, for any pair of boundary points $\underline{x}, \bar{x} \in \partial \Omega$, that

$$
\int_{\gamma} N^{t} \cdot B(x) \cdot N d s=0
$$

where $\gamma$ is the $p$-wave geodesic segment between $\underline{x}$ and $\bar{x}$ with respect to $\left(1 / c_{p}^{2}\right) e$, and

$$
\begin{aligned}
B(x)= & \frac{A_{1}}{c_{p}}-\frac{A_{2}}{c_{p}} \\
= & \kappa\left[\left(\beta_{1}\right)_{x} \otimes\left(\beta_{1}\right)_{x}-\left(\beta_{2}\right)_{x} \otimes\left(\beta_{2}\right)_{x}\right]-\alpha I+\left[\left(\log \sqrt{\frac{\rho_{1}}{\rho_{2}}}\right)_{x} \cdot\right] I \\
& +\left(\log \sqrt{\frac{\rho_{1}}{\rho_{2}}}\right)(s)\left(V+2\left(c_{p}\right)_{x}\right)+2\left(c_{p}^{2}-2 c_{s}^{2}\right)\left(\log \sqrt{\frac{\rho_{1}}{\rho_{2}}}\right)_{x x}, \\
\kappa= & \frac{4 c_{s}^{2}}{c_{p}} \frac{\left(c_{p}^{2}-2 c_{s}^{2}\right)}{\left(c_{p}^{2}-c_{s}^{2}\right)}, \\
\beta_{i}= & \log \sqrt{\rho_{i}}, \quad i=1,2, \\
\alpha= & \frac{\left(c_{p}^{2}-4 c_{s}^{2}\right)}{c_{p}} \Delta\left(\log \sqrt{\frac{\rho_{1}}{\rho_{2}}}\right)-\omega\left[\left.\left(\log \sqrt{\rho_{1}}\right)_{x}\right|^{2}-\left|\left(\log \sqrt{\rho_{2}}\right)_{x}\right|^{2}\right], \\
\omega= & \frac{\left(c_{p}^{2}-4 c_{s}^{2}\right)}{c_{p}}+\frac{4 c_{s}^{4}}{c_{p}\left(c_{p}^{2}-c_{s}^{2}\right)}, \\
V= & 2\left(c_{p}\right)_{x}-\frac{8 c_{s}^{2}}{c_{p}\left(c_{p}^{2}-c_{s}^{2}\right)}\left(c_{s}^{2}\right)_{x} .
\end{aligned}
$$

4.2. Inverting the ray transform. We begin with some preliminary notation, following [Sh II], Sections 2.1-2.4. Let $C^{\infty}\left(\tau_{s}^{r} M\right)$ denote the $r$-times contravariant and $s$-times covariant tensor fields on a manifold $M$. Let $C^{\infty}\left(S^{m} \tau_{M}^{\prime}\right)$ denote the symmetric tensor fields of degree $m$ on $M$, i.e., the $C^{\infty}(M)$-submodule of $C^{\infty}\left(\tau_{m}^{0} M\right)$ that consists of the tensor fields invariant with respect to every transposition of the indices. Let $\sigma: C^{\infty}\left(\tau_{m}^{0} M\right) \rightarrow C^{\infty}\left(S^{m} \tau_{M}^{\prime}\right)$ denote the canonical projection (symmetrization) defined by $\sigma(v)=\frac{1}{m !} \sum_{\pi \in \Pi_{m}} v_{j_{\pi(1)} \ldots j_{\pi(m)}}$, where $\Pi_{m}$ is the group of permutations of the set with $m$ elements.

We define inner differentiation $d: C^{\infty}\left(S^{m} \tau_{M}^{\prime}\right) \rightarrow C^{\infty}\left(S^{m+1} \tau_{M}^{\prime}\right)$ as in [Sh II], Section 2.4, by

$$
d=\sigma \nabla,
$$

where $\nabla$ denotes the Levi-Civita connection associated with the Riemannian manifold $(M, g)$. The divergence operator $\delta: C^{\infty}\left(S^{m+1} \tau_{M}^{\prime}\right) \rightarrow C^{\infty}\left(S^{m} \tau_{M}^{\prime}\right)$ is defined 
in coordinate form by $(\delta v)_{i_{1} \ldots i_{m}}=v_{i_{1} \ldots i_{m} j ; k} g^{j k}$, where $g^{j k}$ is the inverse matrix of the metric tensor $g_{j k}$ on the Riemannian manifold $(M, g)$.

Let $H^{k}\left(S^{m} \tau_{M}^{\prime}\right)$, for any integer $k \geq 0$, be the completion of $C^{\infty}\left(S^{m} \tau_{M}^{\prime}\right)$ with respect to the Sobolev norm $\|\cdot\|_{k}$ corresponding to the scalar product $(\cdot, \cdot)_{k}$ that is defined inductively in $k$ by $(u, v)_{k}=(\nabla u, \nabla v)_{k-1}+(u, v)_{L_{2}}$. In particular, $H^{0}\left(S^{m} \tau_{M}^{\prime}\right)=L_{2}\left(S^{m} \tau_{M}^{\prime}\right)$.

Sharafutdinov shows in Sh II], Theorem 2.4.2, that any symmetric tensor field has a unique decomposition into the sum of "solenoidal" and "potential" parts. That is,

Theorem 4.1 (Sharafutdinov [Sh II]). Let $M$ be a compact Riemannian manifold with boundary. Let $k \geq 1$ and $m \geq 0$ be integers. For every field $f \in H^{k}\left(S^{m} \tau_{M}^{\prime}\right)$, there exist uniquely determined ${ }^{s} f \in H^{k}\left(S^{m} \tau_{M}^{\prime}\right)$ and $v \in H^{k+1}\left(S^{m-1} \tau_{M}^{\prime}\right)$ such that

$$
f={ }^{s} f+d v
$$

where

$$
\delta^{s} f=0 \quad \text { and }\left.\quad v\right|_{\partial M}=0 .
$$

The fields ${ }^{s} f$ and $v$ are called the solenoidal and potential parts of the tensor field $f$.

The ray transform on a CDRM $M$ (cf. notation and terminology introduced in Section 1.1 and in this section) is the linear operator $I: C^{\infty}\left(S^{m} \tau_{M}^{\prime}\right) \rightarrow C^{\infty}\left(\partial_{+} \Omega M\right)$ defined by

$$
I f(x, \xi)=\int_{\tau_{-}(x, \xi)}^{0} f_{i_{1} \ldots i_{m}}\left(\gamma_{x, \xi}(t)\right) \dot{\gamma}_{x, \xi}^{i_{1}}(t) \ldots \dot{\gamma}_{x, \xi}^{i_{m}}(t) d t,
$$

where $\gamma_{x, \xi}:\left[\tau_{-}(x, \xi), 0\right]$ is the maximal geodesic on $M \cup \partial M$ satisfying the initial (i.e., exit) conditions $\gamma_{x, \xi}(0)=x$ and $\dot{\gamma}_{x, \xi}(0)=\xi$.

The ray transform may be inverted, modulo the potential part:

Theorem 4.2 (Sharafutdinov [Sh II] $)$. If $(M, g)$ is a compact, dissipative Riemannian manifold with $(m+1) k^{+}(M, g)<1, m \in\{0,1,2, \ldots\}$, then the solenoidal part sf of any tensor field $f \in H^{1}\left(S^{m} \tau_{M}^{\prime}\right)$ is uniquely determined by its ray transform If.

We define symmetrization with respect to two indices as in [Sh II], in coordinate form, by

$$
\sigma\left(i_{1} i_{2}\right) M_{i_{1} \ldots i_{4}}=\frac{1}{2} \sum_{\pi \in \Pi_{2}} M_{i_{\pi(1)} i_{\pi(2)} i_{3} i_{4}}
$$

where $M$ is a symmetric (here, rank-4) covariant tensor. The Saint-Venant operator $W$ is defined on the space of smooth, symmetric (here, rank-2) tensor fields, mapping into the smooth, symmetric (here, rank-4) tensor fields, by

$$
\begin{aligned}
(W B)_{i_{1} i_{2} j_{1} j_{2}} & =\sigma\left(i_{1} i_{2}\right) \sigma\left(j_{1} j_{2}\right) \sum_{p=0}^{2}(-1)^{p}\left(\begin{array}{l}
2 \\
p
\end{array}\right) B_{i_{1} i_{2-p} j_{1} j_{p}, j_{p+1} j_{2} i_{2-p+1} i_{2}} \\
& =\sigma\left(i_{1} i_{2}\right) \sigma\left(j_{1} j_{2}\right)\left[B_{i_{1} i_{2}, j_{1} j_{2}}-2 B_{i_{1} j_{1}, j_{2} i_{2}}+B_{j_{1} j_{2}, i_{1} i_{2}}\right],
\end{aligned}
$$

where $\left(\begin{array}{l}2 \\ p\end{array}\right)=\frac{2 !}{p !(2-p) !}$ are the binomial coefficients and $B, l$ denotes partial differentiation of $B$ with respect to $x_{l}$. 
Sharafutdinov [Sh I] has shown that the potential parts of the compactly supported, rank-2 symmetric tensor fields $C_{c}^{\infty}\left(S^{2} \tau_{\mathbb{R}^{3}}^{\prime}\right)$ on Euclidean space $\left(\mathbb{R}^{3}, e\right)$ form the kernel of $W$ :

Theorem 4.3 (Sharafutdinov Sh I]). The Saint-Venant operator

$$
W: C_{c}^{\infty}\left(S^{2} \tau_{\mathbb{R}^{3}}^{\prime}\right) \rightarrow C_{c}^{\infty}\left(S^{2} \tau_{\mathbb{R}^{3}}^{\prime} \otimes S^{2} \tau_{\mathbb{R}^{3}}^{\prime}\right)
$$

has kernel

$$
\text { ker } W=\left\{d_{\left(\mathbb{R}^{3}, e\right)} v \mid v \in C^{\infty}\left(S^{1} \tau_{\mathbb{R}^{3}}^{\prime}\right) \text { is compactly supported }\right\} .
$$

Remark 5. We note that $d_{\left(\mathbb{R}^{3}, e\right)} v=\nabla_{x}(s) v$ (cf. notation (30)).

4.3. Proof of Theorem 1. In Section 2 we apply results from $[\mathrm{R} \mathrm{I}$ and $[\mathrm{R} \mathrm{II}$ to show that the wave speeds $c_{p / s}$ are determined in $\Omega$ by the Dirichlet-to-Neumann map. In Section 4.1 we compute the "lower-order polarization" of the elastic wave, and deduce that the ray transform (48) is identically zero if $\Lambda_{\lambda_{1}, \mu_{1}, \rho_{1}}=\Lambda_{\lambda_{2}, \mu_{2}, \rho_{2}}$.

By Theorem 4.1 we decompose the tensor $B$ that appears in the integrand of the ray transform as $B={ }^{s} B+d v$, where $v$ is a rank-1 symmetric tensor field on $\Omega$ and $d$ is the symmetrization of the covariant derivative. By Theorem 4.2 we invert the ray transform (48) to conclude that the solenoidal part ${ }^{s} B$ of $B(x)$ vanishes on $\Omega$. We note that $v$ involves derivatives of $\rho_{i}(x), i=1,2$, of order at most 1 since $B$ involves derivatives of $\rho_{i}$ of order at most 2 . Here we have $(M, g)=\left(\Omega, e / c_{p}^{2}\right)$; so we write $d v=\nabla_{x} S v+R(v)$, where $R(v)$ depends on $v$ and derivatives of $c_{p}$ (cf. notation (30) ). It follows from Theorem 4.3 that $W(B)=W(d v)=W\left(\nabla_{x} S v\right)+W R(v)=$ $W R(v)$ involves derivatives of $\rho_{i}(x)$ of order at most 3 . We write $T_{l} u$ for the sum of terms of $u$ that involve the $l$ th-order derivatives of $\rho_{i}, i=1,2$. Then

$$
T_{4} W(B)=0 .
$$

In the following we derive from this fact a partial differential equation whose solution involves the difference between $\rho_{1}$ and $\rho_{2}$.

We compute the value of $T_{4} W(B)$ acting on certain input. In fact, we first observe (cf. (49) and Theorem 4.3) that

$$
\begin{aligned}
& W\left(\left(\log \sqrt{\rho_{i}}\right)_{x x}\right)=W\left(\nabla_{x} S\left(\log \sqrt{\rho_{i}}\right)_{x}\right)=0, \quad i=1,2, \\
& {[W(\alpha I)]_{i_{1} i_{2} j_{1} j_{2}}=\sigma\left(i_{1} i_{2}\right) \sigma\left(j_{1} j_{2}\right)\left[\delta_{i_{1} i_{2}} \alpha_{, j_{1} j_{2}}-2 \delta_{i_{1} j_{1}} \alpha_{, j_{2} i_{2}}+\delta_{j_{1} j_{2}} \alpha_{, i_{1} i_{2}}\right]} \\
& =\delta_{i_{1} i_{2}} \alpha_{x_{j_{1}} x_{j_{2}}}+\delta_{j_{1} j_{2}} \alpha_{x_{i_{1}} x_{i_{2}}}-\frac{1}{2}\left[\delta_{i_{1} j_{1}} \alpha_{x_{j_{2}} x_{i_{2}}}\right. \\
& \left.+\delta_{i_{2} j_{1}} \alpha_{x_{j_{2} x_{i_{1}}}}+\delta_{i_{1} j_{2}} \alpha_{x_{j_{1}} x_{i_{2}}}+\delta_{i_{2} j_{2}} \alpha_{x_{j_{1}} x_{i_{1}}}\right] \\
& {\left[W\left(\beta_{x} \otimes \beta_{x}\right)\right]_{i_{1} i_{2} j_{1} j_{2}}=\sigma\left(i_{1} i_{2}\right) \sigma\left(j_{1} j_{2}\right)\left[\partial_{x_{j_{2}}} \partial_{x_{j_{1}}}\left(\beta_{x_{i_{1}}} \beta_{x_{i_{2}}}\right)\right.} \\
& \left.-2 \partial_{x_{i_{2}}} \partial_{x_{j_{2}}}\left(\beta_{x_{i_{1}}} \beta_{x_{j_{1}}}\right)+\partial_{x_{i_{2}}} \partial_{x_{i_{1}}}\left(\beta_{x_{j_{1}}} \beta_{x_{j_{2}}}\right)\right] \\
& =\partial_{x_{j_{1}}} \partial_{x_{j_{2}}}\left(\beta_{x_{i_{1}}} \beta_{x_{i_{2}}}\right)+\partial_{x_{i_{1}}} \partial_{x_{i_{2}}}\left(\beta_{x_{j_{1}}} \beta_{x_{j_{2}}}\right) \\
& -2 \sigma\left(i_{1} i_{2}\right) \sigma\left(j_{1} j_{2}\right) \partial_{x_{i_{2}}} \partial_{x_{j_{2}}}\left(\beta_{x_{i_{1}}} \beta_{x_{j_{1}}}\right),
\end{aligned}
$$

where $\delta_{i j}=\left\{\begin{array}{lll}1 & \text { if } & i=j \\ 0 & \text { if } & i \neq j\end{array}\right\}$ is the Kronecker delta. Writing

$$
\varphi^{\prime}=\varphi_{x} \cdot X \quad \text { and } \quad \dot{\varphi}=\varphi_{x} \cdot Y
$$


for any constant vectors $X, Y$ and any smooth scalar function $\varphi$, we find that

$$
\begin{aligned}
W(\alpha I)(X, X, Y, Y) & =|X|^{2} \ddot{\alpha}-2(X \cdot Y) \dot{\alpha}^{\prime}+|Y|^{2} \alpha^{\prime \prime}, \\
W\left(\beta_{x} \otimes \beta_{x}\right)(X, X, Y, Y) & =\left[\left(\beta^{\prime}\right)^{2}\right]^{*}+\left[(\dot{\beta})^{2}\right]^{\prime \prime}-2\left(\dot{\beta} \beta^{\prime}\right)^{. \prime} \\
& =2\left[\dot{\beta}^{\prime} \dot{\beta}^{\prime}-\ddot{\beta} \beta^{\prime \prime}\right] .
\end{aligned}
$$

We choose $X=e_{i}$ and $Y=e_{j}$, where $e_{i}$ is the unit vector in $\mathbb{R}^{3}$ with 1 in the $i$ th component and 0 elsewhere. We sum over $i, j=1,2,3$ to conclude, given (49), that

$$
\begin{aligned}
0= & \sum_{i, j} T_{4} W(B)\left[e_{i}, e_{i}, e_{j}, e_{j}\right] \\
= & T_{4} \sum_{i, j}\left[\kappa W\left(\beta_{1, x} \otimes \beta_{1, x}\right)-\kappa W\left(\beta_{2, x} \otimes \beta_{2, x}\right)-W(\alpha I)\right]\left[e_{i}, e_{i}, e_{j}, e_{j}\right] \\
= & -4 T_{4} \Delta \alpha=-\frac{4\left(c_{p}^{2}-4 c_{s}^{2}\right)}{c_{p}} \Delta^{2}\left(\log \sqrt{\rho_{1}}-\log \sqrt{\rho_{2}}\right) \\
& \quad+4 \omega \Delta\left[\left(\log \sqrt{\rho_{1}}+\log \sqrt{\rho_{2}}\right)_{x} \cdot\left(\log \sqrt{\rho_{1}}-\log \sqrt{\rho_{2}}\right)_{x}\right] .
\end{aligned}
$$

Note that

$$
\omega=\frac{c_{p}^{4}-5 c_{p}^{2} c_{s}^{2}+8 c_{s}^{4}}{c_{p}\left(c_{p}^{2}-c_{s}^{2}\right)}
$$

is never zero on $\bar{\Omega}$, and is bounded on $\bar{\Omega}$, given the strong convexity condition $3 \lambda+2 \mu>0$, i.e., $3 c_{p}^{2}>4 c_{s}^{2}$, on $\bar{\Omega}$. It follows that the difference $\beta^{-}=\log \sqrt{\rho_{1}}-$ $\log \sqrt{\rho_{2}}$ solves the fourth-order, linear, elliptic partial differential equation

$$
\gamma \Delta^{2}\left(\beta^{-}\right)-\Delta\left[\left(\beta^{+}\right)_{x} \cdot\left(\beta^{-}\right)_{x}\right]=0
$$

in $\Omega$, where $\beta^{+}=\beta_{1}+\beta_{2}=\log \sqrt{\rho_{1}}+\log \sqrt{\rho_{2}}$ and

$$
\gamma=\frac{\left(c_{p}^{2}-4 c_{s}^{2}\right) / c_{p}}{\omega}=\frac{\left(c_{p}^{2}-c_{s}^{2}\right)\left(c_{p}^{2}-4 c_{s}^{2}\right)}{c_{p}^{4}-5 c_{p}^{2} c_{s}^{2}+8 c_{s}^{4}} .
$$

Since $c_{p / s}$ are real, $\gamma$ is bounded on $\bar{\Omega}$.

We apply strong uniqueness results for (53) to conclude that $\beta^{-}$vanishes on parts of $\Omega$. In particular, we first note that $\beta^{-}$vanishes to infinite order outside $\partial \Omega$ by Theorem 2.1 and the extension of $\lambda_{j}, \mu_{j}$, and $\rho_{j}$ to $\mathbb{R}^{3}$ (see Section 2.1. We then assume, without loss of generality, that $\lambda_{j}, \mu_{j}$, and $\rho_{j}$ were extended to $\mathbb{R}^{3}$ so that $c_{p} \neq 2 c_{s}$ in a neighborhood of $\partial B_{R}$, where $B_{R} \subseteq \mathbb{R}^{3}$ is an open ball containing $\bar{\Omega}$. Writing

$$
S_{R}=\left\{x \in B_{R}: \gamma=0\right\}=\left\{x \in B_{R}: c_{p}=2 c_{s}\right\}
$$

for the collection of singular points of (53) in $B_{R}$, we conclude that $S_{R}$ does not intersect some neighborhood of $\partial B_{R}$. Let $U_{0}$ be any connected, open neighborhood in $B_{R}$ of $\partial B_{R}$ whose closure has empty intersection with the singular points $S_{R}$. Then $1 / \gamma$ is bounded on $U_{0}$. By (53) and the fact that, outside $\Omega, \beta^{-} \equiv 0$ solves the PDE in (53), we conclude that for any $x_{0} \in U_{0} \backslash \bar{\Omega}$,

$$
\left|\Delta^{2} \beta^{-}\left(x_{0}-x\right)\right| \leq C \sum_{|\alpha| \leq 3}\left|D^{\alpha} \beta^{-}\left(x_{0}-x\right)\right|
$$


on $\left\{x_{0}\right\}-U_{0}$, where $C>0$ is constant. By the strong uniqueness result of $[\mathrm{Pr}$, page 90 , for example, with $u(x)=\beta^{-}\left(x_{0}-x\right)$, it follows that $\beta^{-} \equiv 0$ on $U_{0}$. We vary $U_{0}$ and refer to the continuity of $\rho_{j}$ to conclude that $\beta^{-}=0$ on the closure of the open, connected component $\mathcal{C}_{0}$ of $B_{R} \backslash S_{R}$ that contains a neighborhood in $B_{R}$ of $\partial B_{R}$. That is, $\rho_{1}=\rho_{2}$ on the closure of

$$
\mathcal{C}=\mathcal{C}_{0} \cap \Omega \text {. }
$$

\section{Aknowledgements}

The author would like to thank Todd Quinto, Vladimir Sharafutdinov, and Gunther Uhlmann for many helpful conversations. Thanks, also, to Jeffrey Rauch for suggestions regarding Section 3, and to Rakesh for suggestions regarding Section 4.

\section{REFERENCES}

[De] N. Dencker. On the propagation of singularities for pseudodifferential operators of principal type, Ark. Mat. 20 (1), 1982, 23 - 60. MR 84k:58126

[Eg] Y. V. Egorov. Linear Differential Equations of Principal Type, Contemporary Soviet Math., New York, 1986. MR 88c:35003

[Gr-Sj] A. Grigis and J. Sjöstrand. Microlocal Analysis for Differential Operators, London Math. Soc. Lecture Note Series 196, Cambridge University Press, 1994. MR 95d:35009

[Hö] L. Hörmander. The Analysis of Linear Partial Differential Operators, Vol. I, SpringerVerlag, Berlin, 1983. MR 85g:35002a

[I-N-Y] M. Ikehata, G. Nakamura, and M. Yamamoto. Uniqueness in inverse problems for the isotropic Lamé system, J. Math. Sci. Univ. Tokyo 5 (4), 1998, 627 - 692. MR 2000i:35213

[Pr] M. H. Protter. Unique continuation for elliptic equations, Trans. Amer. Math. Soc. 95 (1), 1960, 81 - 91. MR 22:3871

[R I] L. Rachele. An Inverse Problem in Elastodynamics: Uniqueness of the wave speeds in the interior, J. Diff. Eqs. 162 (2), 2000, 300 - 325. MR 2001e:35177

[R II] L. Rachele. Boundary determination for an inverse problem in elastodynamics, Comm. Partial Diff. Eq. 25 (11-12), 2000, 1951 - 1996. MR 2001m:35322

[R III] L. Rachele. Uniqueness in inverse problems for elastic media with residual stress. To appear in Comm. Partial Diff. Eq.

[Ra-Sy] Rakesh and W. W. Symes. Uniqueness for an inverse problem for the wave equation, Comm. Partial Diff. Eq. 13 (1), 1988, 87 - 96. MR 89f:35208

[Sh I] V. Sharafutdinov. Integral Geometry of Tensor Fields, Inverse and Ill-Posed Problems Series. VSP, Utrecht, 1994. MR 97h:53077

[Sh II] V. Sharafutdinov. Integral geometry of a tensor field on a manifold with upper-bounded curvature, Siberian Math. J. 33 (3), 1992, 524-533. MR 94d:53116

[S-U] J. Sylvester and G. Uhlmann. Inverse problems in anisotropic media. Contemporary Mathematics 122, 1991, 105 - 117. MR 92k:35289

[Tr] F. Treves. Introduction to pseudodifferential and Fourier integral operators, vol. II, Plenum Press, New York, 1980. MR 82i:58068

[U] G. Uhlmann. Inverse boundary value problems and applications, Méthodes semiclassiques, Vol. 1 (Nantes, 1991), Asterisque 207 (6), 1992, 153 - 211. MR 94e:35146

Department of Mathematics, Tufts University, Medford, Massachusetts 02155

Current address: Department of Mathematics and Statistics, University at Albany, SUNY, Albany, New York 12222

E-mail address: lrachele@math.albany.edu 